\title{
SAND98-2476 T
}

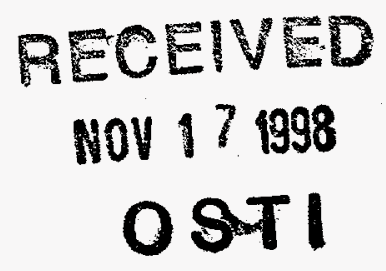

\section{On Theories for Reacting Immiscible Mixtures}

\author{
D. S. Drumheller
}

\begin{abstract}
On some small scale each constituent of an immiscible mixture occupies a separate region of space. Given sufficient time and computing power, we could solve the continuum field equations and boundary conditions for this heterogeneous system. This usually represents an enormously difficult task that is well beyond today's computational capabilities. Mixture theories approximate this complex heterogeneous formulation with a set of field equations for an equivalent homogeneous material. In this work, we compare the theory for immiscible mixtures by Drumheller and Bedford with the theory of Passman, Nunziato, and Walsh. We describe the conditions under which these theories reduce to an equivalent formulation, and we also investigate the differences in their microinertial descriptions.

Two variables play special roles in both theories. They are the true material density and the volume fraction. Here we use a kinematical approach based on two new variables-the true deformation gradient and the distention gradient. We show how the true deformation gradient is connected to the true material density and, in the absence of chemical reactions, the volume fraction is the inverse of the determinant of the distention gradient. However, when chemical reactions occur, the distention gradient and the volume fraction are not directly connected.

We also present a mixture model for a granular explosive. This model is based upon the work of Baer and Nunziato, but our theory differs from their work in that we present a three-dimensiorral model; we cast the constitutive postulates in terms of the distention gradient rather than the volume fraction, and we incorporate elastic-plastic effects into the constitutive description of the solid granules.
\end{abstract}




\section{DISCLAIMER}

This report was prepared as an account of work sponsored by an agency of the United States Government. Neither the United States Government nor any agency thereof, nor any of their employees, make any warranty, express or implied, or assumes any legal liability or responsibility for the accuracy, completeness, or usefulness of any information, apparatus, product, or process disclosed, or represents that its use would not infringe privately owned rights. Reference herein to any specific commercial product, process, or service by trade name, trademark, manufacturer, or otherwise does not necessarily constitute or imply its endorsement, recommendation, or favoring by the United States Government or any agency thereof. The views and opinions of authors expressed herein do not necessarily state or reflect those of the United States Government or any agency thereof. 


\section{DISCLAIMER}

Portions of this document may be illegible in electronic image products. Images are produced from the best available original document. 


\section{Nomenclature}

Symbol Description

$a_{\xi} \quad$ Material acceleration

$\mathbf{A}_{\xi}$ - Distention gradient

$\mathbf{A}_{S}^{E} \quad$ Elastic part of $\mathbf{A}_{S}$

$\mathbf{A}_{S}^{P} \quad$ Plastic part of $\mathbf{A}_{S}$

$\mathbf{b}_{\xi} \quad$ Momentum production rate

$c_{\xi} \quad$ Reaction mass

$c_{\xi}^{+} \quad$ Mass production rate

$d_{S} \quad$ Damage

$\mathcal{D}_{S} \quad$ Damage evolution

$E_{\xi} \quad$ Internal energy

$\dot{e}_{\xi} \quad$ Energy production rate

$e_{\xi}^{+} \quad$ Energy production rate

$\tilde{e}_{S}^{+} \quad$ Energy production rate

$\mathbf{F}_{\xi} \quad$ Deformation gradient

$\overline{\mathbf{F}}_{\xi} \quad$ True deformation gradient

$\overline{\mathbf{F}}_{S}^{E} \quad$ Elastic part of $\overline{\mathbf{F}}_{S}$

$\overline{\mathbf{F}}_{S}^{P} \quad$ Plastic part of $\overline{\mathbf{F}}_{S}$

$\mathbf{f}_{\xi} \quad$ Body force

$\mathbf{G}_{\xi} \quad$ Temperature gradient

$\mathcal{G}_{\xi} \quad$ Gibb's energy

$H_{\xi} \quad$ Enthalpy

$h \quad$ Heat transfer coefficient

$I_{\xi} \quad$ Microinertia

$J_{\xi} \quad$ Jacobian of $\mathbf{F}_{\xi}$

$\bar{J}_{\xi} \quad$ Jacobian of $\overline{\mathbf{F}}_{\xi}$

$k_{\xi} \quad$ Microinertia

$\mathcal{L}_{\xi} \quad$ Velocity gradient

$L_{\xi} \quad$ Generalized force

$\mathbf{m}_{S}^{+} \quad$ Momentum production rate

$\tilde{\mathbf{m}}_{S}^{+} \quad$ Momentum production rate

$N \quad$ Number of constituents

$p_{\xi} \quad$ Pressure - . -
Defining Equation

(48) (50)

(52) 


\begin{tabular}{cl} 
Symbol & Description \\
\hline & \\
$p_{S e}$ & Equilibrium $p_{S}$ \\
$\bar{p}_{S e}$ & Equilibrium true pressure \\
$\mathbf{g}_{\xi}$ & Heat flux \\
$r_{\xi}$ & External heat supply \\
$R$ & Bubble radius \\
$\mathbf{R}_{S}$ & Distention rotation \\
$t$ & Time \\
)$^{t}$ & Transpose operator \\
$\mathbf{T}_{\xi}$ & Stress \\
$\mathbf{T}_{S}^{\prime}$ & Deviatoric stress \\
$\mathbf{T}_{S e}$ & Equilibrium $\mathbf{T}_{S}$ \\
$\overline{\mathbf{T}}_{R e}$ & Equilibrium stress of reference solid \\
$\overline{\mathbf{T}}_{S e}$ & Equilibrium true stress \\
$\mathbf{u}_{\xi}$ & Diffusion velocity \\
$\mathbf{v}$ & Barycentric velocity \\
$\mathbf{v}_{\xi}$ & Material velocity \\
$\mathbf{x}$ & Material position \\
$\overline{\mathbf{x}}_{\xi}$ & True material position \\
$\mathbf{X}_{\xi}$ & Reference position \\
$Y^{A}$ & Yield function (distention) \\
$Y_{F}^{F}$ & Yield function (true motion) \\
$\alpha_{\xi}$ & Distention \\
$\alpha_{S}^{E}$ & Elastic distention \\
$\alpha_{S}^{P}$ & Plastic distention \\
$\beta_{S}$ & Pressure \\
$\beta_{S e}$ & Equilibrium $\beta_{S}$ \\
$\delta$ & Drag coefficient \\
$\eta_{\xi}$ & Entropy \\
$\vartheta_{\xi}$ & Temperature \\
$\Lambda_{\xi}$ & Primitive variable set \\
$\lambda$ & Interface pressure \\
$\mu_{\xi}$ & Lagrange multiplier \\
$\nu_{A}$ & Compaction parameter \\
$\nu_{G}$ & Compaction parameter \\
$\rho$ & Mixture density \\
& \\
\hline
\end{tabular}

Defining Equation 


\begin{tabular}{clc} 
Symbol & Description & Defining Equation \\
\hline & & $(4)$ \\
$\rho_{\xi}$ & Partial density & $(29)$ \\
$\rho_{\xi 0}$ & Reference value of $\rho_{\xi}$ & $(5)$ \\
$\bar{\rho}_{\xi}$ & True density & $(34)$ \\
$\bar{\rho}_{\xi 0}$ & Reference value of $\bar{\rho}_{\xi}$ & $(44)$ \\
$\tau$ & Lagrange multiplier & $(1)$ \\
$\varphi_{\xi}$ & Volume fraction & $(34)$ \\
$\varphi_{\xi 0}$ & Reference value of $\varphi_{\xi}$ & $(11)$ \\
$\chi_{\xi}$ & Material motion & $(14)$ \\
$\hat{\chi}_{\xi}$ & Distention motion & $(15)$ \\
$\tilde{\chi}_{\xi}$ & True motion & $(69)$ \\
$\psi_{\xi}$ & Helmholtz free energy & $(25)(26)(43)$ \\
()$_{\mathbf{x}_{\gamma}}$ & Material derivative operator
\end{tabular}




\section{Introduction}

The theory of mixtures has undergone significant developments in recent decades. For example see the review article by Bedford and Drumheller [1] and the book by Truesdell [2]. People in continuum mechanics place most mixtures into one of two general categorieseither immiscible or miscible mixtures. The theories for immiscible mixtures employ the concept of volume fraction, which will be defined below, whereas theories for miscible mixtures do not employ this variable. Blood is an example of an immiscible mixture of cells and plasma, while in contrast, air is a miscible mixture of nitrogen and oxygen with small amounts of argon, carbon dioxide, and hydrogen. Sometimes the term multiphase mixture is applied to an immiscible mixture of two phases of the same material such as in boiling water. Continuum theories have been designed for both miscible and immiscible mixtures. In these theories, we assume that each constituent simultaneously occupies the same region of space. Of course on some small spatial scale especially in the case of immiscible mixtures this is not true, but this assumption is made to facilitate the development of tractable continuum theories that still retain what we might judge to be the essential features of the mixture. As we shall show, such judgments are often very subjective.

Our topic is immiscible mixtures and more specifically on the role that volume fraction has played in the development of these theories. We will assume the mixture is composed of $N$ constituents. When we speak of a particular constituent we will usually refer to it as the $\xi$-th constituent of the mixture, where any lower-case Greek letter might be used. But we will also pay special attention to a binary mixture, $N=2$, which consists of a porous solid, $\xi=S$, and a gas, $\xi=G$. This class of binary mixture has been used to model the burning and detonation of propellants and explosives [3]. In this special case the stress in the solid is represented by a bold-faced symbol $\mathbf{T}_{S}$ because it is a second order tensor, while the pressure in the gas is represented by an italic symbol $p_{G}$ because it is a scalar.

As we mentioned above, the crucial parameter that distinguishes a theory for immiscible mixtures from a theory for miscible, mixtures is the scalar quantity called the volume fraction, $\varphi_{\xi}$. For any material volume $d V$ of the mixture, a portion of that volume $d V_{\xi}$ is occupied 
only by the $\xi$-th constituent. The volume fraction $\varphi_{\xi}$ is given by

$$
\varphi_{\xi}=d V_{\xi} / d V
$$

An immiscible mixture is defined to be saturated if

$$
d V=\sum_{\xi} d V_{\xi}
$$

which implies that

$$
\sum_{\xi} \varphi_{\xi}=1
$$

Let the mass of the $\xi$-th constituent contained in $d V$ be $d m_{\xi}$. The partial density $\rho_{\xi}$ is defined to be

$$
\rho_{\xi} \equiv d m_{\xi} / d V
$$

and the true density $\bar{\rho}_{\xi}$ is defined to be

$$
\bar{\rho}_{\xi} \equiv d m_{\xi} / d V_{\xi}
$$

The definition of volume fraction Eq. (1) then leads to the relation

$$
\rho_{\xi}=\varphi_{\xi} \bar{\rho}_{\xi} .
$$

In the 1983 review article by Bedford and the author [1], we discussed the historical development of the volume fraction concept. We listed numerous references on this particular issue, which go back to 1966. One of the key points of contention in these early works was the form of the equations of balance of momentum for a mixture of fluids. Two possibilities existed for the $\xi$-th constituent. When terms that represented the momentum interaction between the constituents were ignored, these two possibilities gave either

$$
\rho_{\xi} \mathbf{a}_{\xi}=-\operatorname{grad}\left(\varphi_{\xi} p_{\xi}\right)
$$

or

$$
\rho_{\xi} \mathbf{a}_{\xi}=-\varphi_{\xi} \operatorname{grad} p_{\xi}
$$

where $\mathbf{a}_{\xi}$ is the acceleration of the $\xi$-th constituent. Equation $(7)$; which states that the motion is driven by the gradient of the partial pressure $\varphi_{\xi} p_{\xi}$, is the classical result for miscible mixtures. However, this form seemed inappropriate for immiscible mixtures. In contrast, 
Eq. (8) seemed more appropriate for immiscible mixtures because division by $\varphi_{\xi}$ yielded

$$
\bar{\rho}_{\xi} \mathbf{a}_{\xi}=-\operatorname{grad} p_{\xi} .
$$

This is the "true" balance of momentum for the $\xi$-th constituent, and as the review article pointed out, recovery of this result was crucial to any immiscible mixture theory. To see why, let us consider the difference between these two forms of the balance of momentum, which is

$$
p_{\xi} \operatorname{grad} \varphi_{\xi} .
$$

This is often called the buoyancy term, and it appears in Eq. (7) but not in Eq. (8). The reason for the choice of this name is not totally clear, but the following specific example may shed light on this terminology as well as illustrate an important contradiction caused by this term.

Consider a bed of sand at rest. If the grains of sand are not packed uniformly in this bed, $\operatorname{grad} \varphi_{\xi} \neq \mathbf{0}$. Ignoring gravitational effects, the sand should remain stationary if $p_{\xi}=$ constant. The presence of the buoyancy term in Eq. (7) causes it to predict motion of the sand and water for this example while Eq. (8) does not.

In a 1978 paper [4], Bedford and the author first showed how this point of contention could be resolved by application of the volume fraction constraint Eq.(3). We derived a mechanical theory for a mixture of immiscible fluids using a form of Hamilton's variational principle, which allowed us to enforce the volume fraction constraint with the method of Lagrange multipliers (see Bedford [5]). The multiplier condition produced a new variable we called the interface pressure. At a later date, we extended our theory to include mixtures of solids and fluids with chemical reactions and diffusion (see [6]). Using Hamilton's principle allowed us to include not only the volume fraction constraint but also constraints on the mass transfer during chemical reactions between constituents. Concurrently with [6], Nunziato and Walsh [7] presented a similar theory in which the constraint conditions were not introduced into the balance of momentum postulates but instead were introduced at a later stage of the derivation during their treatment of the entropy inequality. This type of developmertsmplies that the interface pressure is in some way directly associated with dissipation; however, from our earlier work it is clear that this is not so.

The work of Bedford and the author [6] as well as that of Nunziato 
and Walsh [7] form the basis of most modern theories for immiscible mixtures. These are the two works that we shall discuss here. In addition, we shall also refer to a summary article by Passman, Nunziato, and Walsh [8]. For simplicity we shall refer to [6], [7], and [8] as the primary references. There are subtle differences in the two theories, which we shall point out. However, in many respects they are quite similar. Is it also true that you can find numerous applications of these two theories to experimental data reported in the references we cite. Here our goal is not to report additional comparisons to data, but rather we will address a more fundamental issue. This issue concerns a more precise description of volume fraction in terms of the kinematics of these theories. Let us now discuss why this issue is so important.

In the primary references the quantity volume fraction $\varphi_{\xi}$ is postulated much in the same way we have introduced it here. It is not directly connected to the material motion. Indeed this has lead to subjective interpretations of the precise relationship between the material motion and the volume fraction variable. For example, Passman, Nunziato, and Walsh [8] argued that changes in $\varphi_{\xi}$ represent material motions that should be governed by an additional balance of momentum. In essence they claimed that volume fraction is a "kinematical variable." Thus they postulated additional equilibrated inertia equations to describe these microscale motions. In contrast, Drumheller and Bedford [6] chose to write a balance equation for $\bar{\rho}_{\xi}$ rather than for $\varphi_{\xi}$. Both choices are subjective because neither theory demonstrates a precise relationship between the volume fraction and the material motion.

Here we shall resolve this issue by presenting a kinematical description that allows the volume fraction to be derived from the postulates for the material motion. As you might expect, changing something as fundamental as the description of motion in any theory has the potential to alter entirely the structure of that theory. However, we shall demonstrate how this new description can be fit into the framework of the existing formulations and exactly how it resolves many of the key differences in the primary references. Indeed, to demonstrate just how important this topic is, let us illustrate the essence of a problem. faced by the two theories in their present forms. In particular, this example illustrates that the volume fraction can change in the absence of motion, and thus in this example it is not a kinematical variable. 
Let us consider the simple example illustrated in Figure 1. Two

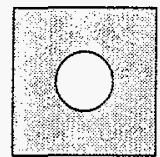

$\bar{\rho}_{\text {so, }} \varphi_{\text {so }}$

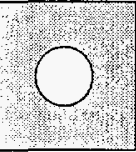

$\bar{\rho}_{s o}, \varphi_{s o}$

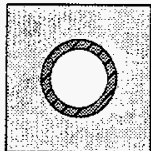

Burn

BLOCK A

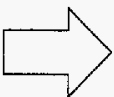

Stretch

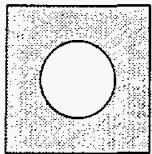

$\bar{\rho}_{s 0}, \varphi_{S}$

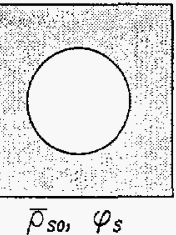

BLOCK B

Figure 1: The effect of burning on volume fraction.

identical solid blocks $\mathrm{A}$ and $\mathrm{B}$ are shown. Each contains a hole. These blocks represent idealized elemental volumes of a porous solid $(\xi=S)$. From left to right, Block $A$ is shown in an initial, intermediate, and final state. Block $B$ is shown in an initial and final state. On the left, the true density and volume fraction of Block $A$ are $\left(\bar{\rho}_{S 0}, \varphi_{S 0}\right)$. We change the size of the hole in block $A$ by burning away a shell of solid material. We do not allow any motion of the solid, and thus the true density of the solid cannot change, but obviously the volume fraction does change. The final values of these quantities at the state on the right are $\left(\bar{\rho}_{S 0}, \varphi_{S}\right)$. Next we change the volume fraction of block $B$ by stretching it. We do not allow burning and we do not allow the true density to change. When we change the volume fraction of block $B$ to match the final volume fraction of block $A$, we notice that the final volume fractions and true densities of the two blocks are equal, but the processes that got the two blocks to these states are quite different. For Block $A$ the change in volume fraction is not accompanied by 
motion. For Block B it is accompanied by motion. Obviously neither the volume fraction nor the true density describe the micromotion that occurs in this example.

There is also a more subtle issue illustrated here. Suppose that we want to construct a constitutive description for a porous solid composed of these elemental blocks. If, as is sometimes assumed, our constitutive description depends only on the true density $\bar{\rho}_{S}$ and the volume fraction $\varphi_{S}$, then the constitutive description cannot distinguish between the two different processes followed by blocks $\mathrm{A}$ and $\mathrm{B}$. We have no way of determining if the material was either stretched or subjected to burning. The theory of Baer and Nunziato [3], which describes granular explosives, is of this type because the Helmholtz free energy of the granular solid depends only on the true density and the volume fraction of the solid constituent. Such a description leads to an unsatisfactory characterization of the material because it requires that the free energies of Blocks $A$ and $B$ must be equal when their temperatures are equal. Let us now turn to a kinematical description that resolves these dilemmas.

\section{$3 \quad$ Kinematics}

In the primary references the kinematics of immiscible mixtures are formulated in terms of postulates for the motion and the volume fraction $\varphi_{\xi}$. There is no distinction between the portion of the motion that causes the volume fraction to change and the portion of the motion that does not affect the volume fraction. Here we make a clear distinction between these two types of motion.

The motion of each constituent of the mixture is given by

$$
\mathbf{x}=\chi_{\xi}\left(\mathbf{X}_{\xi}, t\right)
$$

where $\mathbf{X}_{\xi}$ is the reference position and $t$ is the time. The velocity $\mathbf{v}_{\xi}$ of the $\xi$-th constituent is then

$$
\mathbf{v}_{\xi} \equiv \frac{\partial}{\partial t} \chi_{\xi}\left(\mathbf{X}_{\xi}, t\right)
$$

and the acceleration $\mathbf{a}_{\xi}$ is

$$
\mathbf{a}_{\xi} \equiv \frac{\partial}{\partial t} \mathbf{v}_{\xi}\left(\mathbf{X}_{\xi}, t\right)
$$


While the primary references build theories only upon the basis of the motion Eq. (11) and the volume fraction Eq. (1), here we shall add another step and decompose the motion of each constituent Eq. (11) as follows: ${ }^{1}$

$$
\begin{aligned}
\mathbf{x} & =\hat{\chi}_{\xi}\left(\overline{\mathbf{x}}_{\xi}, t\right) \\
\overline{\mathbf{x}}_{\xi} & =\breve{\chi}_{\xi}\left(\mathbf{X}_{\xi}, t\right) .
\end{aligned}
$$

We call $\breve{\chi}_{\xi}\left(\mathbf{X}_{\xi}, t\right)$ the true motion and $\hat{\chi}_{\xi}\left(\overline{\mathbf{x}}_{\xi}, t\right)$ the distention motion. The reason for this nomenclature will soon become apparent.

The deformation gradient of the $\xi$-th constituent is defined as

$$
\mathbf{F}_{\xi} \equiv \frac{\partial \chi_{\xi}}{\partial \mathbf{X}_{\xi}}
$$

We also find that

$$
\mathbf{F}_{\xi}=\mathbf{A}_{\xi} \overline{\mathbf{F}}_{\xi}
$$

where

$$
\begin{aligned}
& \mathbf{A}_{\xi} \equiv \frac{\partial \hat{\chi}_{\xi}}{\partial \overline{\mathbf{x}}_{\xi}} \\
& \overline{\mathbf{F}}_{\xi} \equiv \frac{\partial \breve{\chi}_{\xi}}{\partial \mathbf{X}_{\xi}} .
\end{aligned}
$$

Here $\mathbf{A}_{\xi}$ is the distention gradient of the $\xi$-th constituent and $\overline{\mathbf{F}}_{\xi}$ is the true deformation gradient. This type of decomposition of the motion was suggested by Davison, Stevens, and Kipp in 1977, [11]. Drumheller [12] discussed a decomposition of this type for elasticplastic effects. Also, De Boer and Bluhm [13] investigated the kinematics of mixtures using a nearly identical decomposition of the motion; however, they omitted chemical reactions and, as we shall see, missed an opportunity to investigate how the volume fraction decouples from the motion. ${ }^{2}$

\footnotetext{
${ }^{1}$ In the past, the additive decomposition of motion has often been employed to model materials with microstructure. For examples see Mindlin [9] and Eringen [10]. The resulting theories bear little resemblance to the mixture theories discussed here.

${ }^{2}$ The decomposition that we have chosen is not unique. For example, we might have decomposed the motion so that instead of Eq. (17) we would have obtained

$$
\mathbf{F}_{\xi}=\overline{\mathbf{F}}_{\xi} \mathbf{A}_{\xi}
$$
}


The Jacobians of the motion and the true motion are

$$
\begin{aligned}
J_{\xi} & \equiv \operatorname{det} \mathbf{F}_{\xi}, \\
\bar{J}_{\xi} & \equiv \operatorname{det} \overline{\mathbf{F}}_{\xi}
\end{aligned}
$$

We require that $J_{\xi}>0$ and $\bar{J}_{\xi}>0$, which insures that the inverse of the respective motions and their deformation gradients exist. Next we define the distention $\alpha_{\xi}$ of the $\xi$-th constituent to be

$$
\alpha_{\xi}=\operatorname{det} \mathbf{A}_{\xi}
$$

Thus

$$
J_{\xi}=\alpha_{\xi} \bar{J}_{\xi}
$$

and we also find that $\alpha_{\xi}>0$. Now we recall that for any arbitrary tensor B

$$
\frac{\partial}{\partial B_{i j}} \operatorname{det} \mathbf{B}=\operatorname{cof} B_{i j}
$$

where $\operatorname{cof} B_{i j}$ denotes the cofactor of the matrix of the elements of $\mathbf{B}$. Moreover the total material derivative of an arbitrary function $\Gamma_{\xi}(\mathbf{x}, t)$ following the motion of the $\gamma$-th constituent is

$$
\dot{\Gamma}_{\xi} \mid \mathbf{x}_{\gamma} \equiv \frac{\partial}{\partial t} \Gamma_{\xi}+\mathbf{v}_{\gamma} \cdot \operatorname{grad} \Gamma_{\xi}
$$

To simplify notation we let

$$
\dot{\Gamma}_{\xi} \equiv \dot{\Gamma}_{\xi} \mid \mathbf{x}_{\xi}
$$

Using these results we find that

$$
\dot{J}_{\xi}=J_{\xi} \operatorname{div} \mathbf{v}_{\xi},
$$

and the velocity gradient $\mathcal{L}_{\xi}$ is

$$
\mathcal{L}_{\xi} \equiv \frac{\partial}{\partial \mathbf{x}} \mathbf{v}_{\xi}(\mathbf{x}, t)=\dot{\mathbf{F}}_{\xi} \mathbf{F}_{\xi}^{-1}
$$

Because the tensor product is not commutative, these values $\overline{\mathbf{F}}_{\xi}$ and $\mathbf{A}_{\xi}$ are different from those in Eq. (17) unless one of them is spherical. This is an old issue that has raised many discussions particularly in the field of plasticity where the motion is decomposed into elastic and plastic parts [12]. It is a moot point because each choice leads to different definitions of stress that automatically compensate for the different decompositions. 


\section{Conservation of Mass}

In the previous section we partitioned the motion into true motion and distention motion. So far the nomenclature associated with these parts of motion is completely arbitrary. In this section we assign the properties to each of the parts of motion that distinguish one type of motion from the other and provides the motivation for our choice of nomenclature.

The primary references employ equivalent statements for the conservation of mass. To illustrate this, let us begin with [6], which states that the conservation of mass for the $\xi$-th constituent is

$$
\rho_{\xi} J_{\xi}=\rho_{\xi 0}+c_{\xi}
$$

Here $\rho_{\xi 0}$ is the partial density of the material in the reference configuration and $c_{\xi}$ is the mass contribution to the reference configuration due to mass transfer from other constituents. Taking the total material derivative of this equation and substituting Eq. (27) yields

$$
\dot{\rho}_{\xi}+\rho_{\xi} \operatorname{div} \mathbf{v}_{\xi}=c_{\xi}^{+}
$$

where

$$
c_{\xi}^{+} \equiv \dot{c}_{\xi} / J_{\xi}
$$

This form of the equation of the conservation of mass appears in each of the primary references. These references also show that

$$
\sum_{\xi} c_{\xi}^{+}=0
$$

is the equation of conservation of mass for the entire mixture. Combining Eqs. (6) and (23) with Eq. (29), we obtain

$$
\varphi_{\xi} \bar{\rho}_{\xi} \bar{J}_{\xi} \alpha_{\xi}=\varphi_{\xi 0} \bar{\rho}_{\xi 0}+c_{\xi}
$$

where the volume fraction $\varphi_{\xi 0}$ and the true density $\bar{\rho}_{\xi 0}$ of the reference material obey

$$
\rho_{\xi 0}=\varphi_{\xi 0} \bar{\rho}_{\xi 0} .
$$

Now we are at a juncture where we can tie the new kinematical basis we presented in the previous section to the mixture formalism as derived in the primary references. This will allow us to establish a 
connection between the distention and the volume fraction. To make this connection we require that

$$
\bar{\rho}_{\xi} \bar{J}_{\xi}=\bar{\rho}_{\xi 0}
$$

We call-this the true conservation of mass, because it resembles the classical conservation of mass for a single material. When it is substituted into Eq. (33), we obtain

$$
\varphi_{\xi} \alpha_{\xi}=\varphi_{\xi 0}+\frac{c_{\xi}}{\bar{\rho}_{\xi 0}}
$$

This is a key result of this work. It demonstrates that volume fraction is related directly to the motion only in the absence of mass exchange. ${ }^{3}$

The derivative of the logarithm of this expression yields

$$
\frac{\dot{\alpha}_{\xi}}{\alpha_{\xi}}=\frac{c_{\xi}^{+}}{\rho_{\xi}}-\frac{\dot{\varphi}_{\xi}}{\varphi_{\xi}}=\frac{\dot{\bar{\rho}}_{\xi}}{\bar{\rho}_{\xi}}+\operatorname{div} \mathrm{v}_{\xi}
$$

The equation of conservation of mass for the entire mixture is given by Eq. (32), but this conservation law can also be expressed in another way. We first define the mixture mass density $\rho$

$$
\rho \equiv \sum_{\xi} \rho_{\xi}
$$

and the barycentric or mixture velocity $\mathbf{v}$

$$
\rho \mathbf{v} \equiv \sum_{\xi} \rho_{\xi} \mathbf{v}_{\xi}
$$

Notice that the diffusion velocity $\mathbf{u}_{\xi}$ can now be defined as

$$
\mathbf{u}_{\xi} \equiv \mathbf{v}_{\xi}-\mathbf{v}
$$

Summation of Eq. (30) over all values of $\xi$ and substitution of Eq. (32) yields

$$
\dot{\rho}+\rho \operatorname{div} \mathrm{v}=0
$$

\footnotetext{
${ }^{3}$ When mass exchange is omitted from Eq. (36) and we assume that $\varphi_{\xi 0}=1$, then the distention is simply the inverse of the volume fraction. This is the classical definition of distention given by $W$. Herrmann in his 1969 paper on the dynamic compaction of porous materials [14].
} 
where

$$
\dot{\rho}=\frac{\partial}{\partial t} \rho+\mathbf{v} \cdot \operatorname{grad} \rho .
$$

Variables such as $\rho(\mathbf{x}, t)$ that are not associated with a particular constituent do not have a Greek subscript. The material derivative of these variables follows the barycentric motion $\mathbf{v}$. Thus for an arbitrary variable $\Gamma(\mathbf{x}, t)$

$$
\dot{\Gamma} \equiv \frac{\partial}{\partial t} \Gamma+\mathbf{v} \cdot \operatorname{grad} \Gamma
$$

What we have shown in this and the previous section is that volume fraction and true density are linked to the motion through the true deformation gradient $\overline{\mathbf{F}}_{\xi}$, the distention gradient $\mathbf{A}_{\xi}$, and the mass exchange $c_{\xi}$. The true deformation gradient $\overline{\mathbf{F}}_{\xi}$ is uniquely connected to the true density $\bar{\rho}_{\xi}$ through the true conservation of mass Eq. (35); however, the distention gradient $\mathbf{A}_{\xi}$ is connected to both the volume fraction $\varphi_{\xi}$ and the mass exchange $c_{\xi}$. Previous works have not established these clear links between the motion and the variables $\bar{\rho}_{\xi}, \varphi_{\xi}$, and either $c_{\xi}$ or $c_{\xi}^{+}$.

What advantages do the definitions of distention motion $\hat{\chi}_{\xi}$, distention gradient $\mathbf{A}_{\xi}$, and the distention $\alpha_{\xi}$ offer to the theories of immiscible of mixtures? To answer this question let us return to the example illustrated with Blocks $A$ and $B$ in Figure 1. We have illustrated these blocks again in Figure 2, but this time we have also shown how the processes of burning Block A and stretching Block B have altered the distention. We recall that burning does not change the motion. Thus the distention of Block $\mathrm{A}$ is not affected by burning; however, because the true density is held constant during stretching, the distention of Block B is changed. Thus by using distention we can distinguish between the burning process acting on Block $A$ and the stretching process acting on Block $\mathrm{B}$.

As we have pointed out, Passman, Nunziato, and Walsh [8] have argued that the true density $\bar{\rho}_{\xi}$ and the volume fraction $\varphi_{\xi}$ are distinctly different variables, they suggested that $\varphi_{\xi}$ is "recognized ... as a kinematical variable and (should) be governed by an equation of balance." (See [8], page 287.) Here we shall call this a microinertia equation. They also believed that a similar microinertia equation for $\bar{\rho}_{\xi}$ was not necessary. Consequently they believed that $\varphi_{\xi}$ is a kinematical variable and $\bar{\rho}_{\xi}$ is not. This is in direct conflict with the present derivation. We shall return to this issue later. In contrast, 


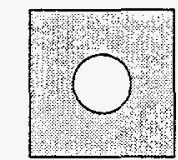

$\bar{\rho}_{\text {so }} \varphi_{\text {so }}, \alpha_{\text {so }}$

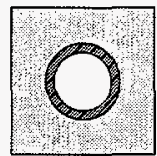

Burn

BLOCK A

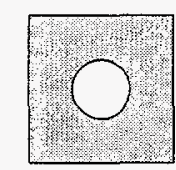

$\bar{\rho}_{\text {so }}, \varphi_{\text {so }}, \alpha_{\text {so }}$

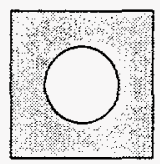

$\bar{\rho}_{\text {so, }} \varphi_{s}, \alpha_{\text {so }}$

$$
\text { A }
$$

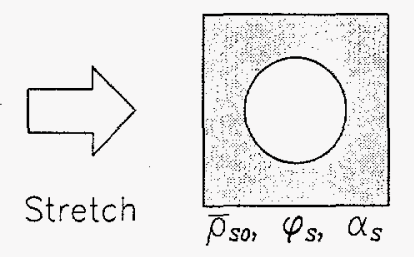

BLOCK B

Figure 2: The effect of burning on volume fraction and distention.

Bedford and the author were skeptical of this approach and instead postulated a microinertia equation for $\bar{\rho}_{\xi}$, which implies that $\bar{\rho}_{\xi}$ is indeed a kinematic variable. In later work (see Appendix $C$ of [1]), we also suggested that microinertia equations could be written for both $\bar{\rho}_{\xi}$ and $\varphi_{\xi}$.

Finally the work of Baer and Nunziato indeed implies that microinertia equations are more properly written in terms of distention rather than volume fraction. We shall examine their model for granular explosives in greater detail in the latter part of this work. ${ }^{4}$

\footnotetext{
${ }^{4}$ In a private communication M. R. Baer stated that an argument similar to the example in this section influenced the construction of his one-dimensional constitutive theory for granular explosives (see Baer and Nunziato [3]). That theory contains a constitutive equation called a closure condition that indeed is a constitutive equation for the rate of change of the distention; however, as we have already pointed out, the distention was omitted from the rest of the constitutive description and most importantly from the Helmholtz free energy for the unreacted solid explosive.
} 


\section{Balance of Momentum}

We have already shown that the different postulates of conservation of mass given in the primary references are equivalent. The primary references also contain equivalent equations of balance of momentum and conservation of energy; however, demonstrating this equivalence is more involved. In this section and the next we state the equations of balance of momentum and conservation of energy from [6]. Following this we reduce these results to obtain the equations in [8].

In [6] we derived the balance of momentum from a postulated form of Hamilton's principle (see also Bedford [5]). We stated Hamilton's principle in terms of pairs of conjugate variables. ${ }^{5}$ For the $\xi$-th constituent, we consider two sets of conjugate variables. We suppose that a stress $\mathbf{T}_{\boldsymbol{\xi}}$ exists that does work when the deformation gradient $\mathbf{F}_{\boldsymbol{\xi}}$ changes, and because of the partitioning of the motion of the constituents by Eq. (14), we also realize that a pressure $p_{\xi}$ can do work on the constituent as $\bar{\rho}_{\xi}$ changes even while $\mathbf{F}_{\xi}$ is held fixed. ${ }^{6}$ In [6] we employed the method of Lagrange multipliers to introduce several constraints into the theory. The volume fraction constraint Eq. (3), the conservation of mass Eq. (29), and the mixture conservation of mass Eq. (32) were explicitly included by this method. Thus in [6] we derive the following equation for the balance of momentum of the $\xi$-th constituent:

$\rho_{\xi} \mathbf{a}_{\xi}=\operatorname{div} \mathbf{T}_{\xi}+\rho_{\xi} \mathbf{f}_{\xi}+\mathbf{b}_{\xi}+\lambda \operatorname{grad} \varphi_{\xi}-\operatorname{grad}\left(\mu_{\xi} J_{\xi}\right)+c_{\xi}^{+}\left(\operatorname{grad} \tau-\mathbf{v}_{\xi}\right)$.

The first two terms on the right-hand side of this equation are conventional expressions. They are the divergence of the stress tensor $\mathbf{T}_{\xi}$ and the external body force vector $\mathbf{f}_{\xi}$. The remaining terms are special to mixture theory. Of these the third term is the momentum production vector $\mathbf{b}_{\xi}$, which is the contribution to the momentum of the $\xi$-th constituent through interaction with its neighboring constituents. The last three terms each contain a different Lagrange multiplier. The term

\footnotetext{
${ }^{5}$ Conjugate variable pairs contain a "flux" variable, such as rate of change of density, and a "force variable", such as pressure. Each product of these individual pairs contributes to the rate of change of energy. Truesdell [2] has an excellent discussion of how conjugate pairs can be used to define physical components in continuum field theories.

${ }^{6}$ We might also argue that rather than the scalar $p_{\xi}$ another stress tensor exists that does work as $\overline{\mathbf{F}}_{\xi}$ changes. This is the topic of a forthcoming work.
} 
with the multiplier $\lambda$ enforces the volume fraction constraint Eq. (3), the multiplier $\mu_{\xi}$ enforces the conservation of mass Eq. (29), and the multiplier $\tau$ enforces the mixture conservation of mass Eq. (32). The significance of these multipliers will become clear as we proceed. For example, we will show that $\lambda$ is the interface pressure acting between the constituents.

The primary references also list microinertia equations. While microinertia is discussed in greater detail in the appendix, here we shall ignore the contributions of microinertia, and therefore [6] gives the following relationships between the constituent pressure and the Lagrange multipliers:

$$
\begin{aligned}
& \frac{\mu_{\xi} J_{\xi}}{\varphi_{\xi}}=p_{\xi}, \\
& \frac{\mu_{\xi} J_{\xi}}{\varphi_{\xi}}=\lambda,
\end{aligned}
$$

which yield

$$
p_{\xi}=\lambda \text {. }
$$

This means that the pressures $p_{\xi}$ for all of the constituents are equal. You should notice that the $p_{\xi}$ are not necessarily equilibrium pressures. Theories in which the constituents are all required to have identical equilibrium pressures often yield ill-posed mathematical formulations. (For example, see Embid and Baer [16].) Accompanying these relationships is another balance equation controlling the exchange of kinetic energy and work during an exchange of mass between constituents. It is

$$
\frac{1}{2} \mathbf{v}_{\xi} \cdot \mathbf{v}_{\xi}+L_{\xi}-\frac{\mu_{\xi} J_{\xi}}{\rho_{\xi}}=\dot{\tau} \mid \mathbf{x}_{\xi} \cdot
$$

We call $L_{\xi}$ a generalized force that acts on the exchange of mass. We shall see that $L_{\xi}$ as well as $\tau$ can be explicitly removed from the field equations.

The equation of balance of momentum for the entire mixture is

$$
\sum_{\xi} \mathbf{b}_{\xi}=\mathbf{0}
$$

The equations in this section are the equations of balance of momentum given in [6]. We shall show that they reduce to the equations of balance of momentum given in [8] but first we will discuss the equations of conservation of energy. 


\section{Conservation of Energy}

The conservation of energy postulates used by Bedford and the author [6] are

$$
\rho_{\xi} \dot{E}_{\xi}+c_{\xi}^{+} E_{\xi}=\operatorname{tr}\left(\mathbf{T}_{\xi}^{t} \mathcal{L}_{\xi}\right)+\varphi_{\xi} p_{\xi} \frac{\dot{\bar{\rho}}_{\xi}}{\bar{\rho}_{\xi}}-L_{\xi} c_{\xi}^{+}-\operatorname{div} \mathbf{q}_{\xi}+\rho_{\xi} r_{\xi}+\dot{e}_{\xi}
$$

where $E_{\xi}$ is the specific internal energy, $r_{\xi}$ is the external heat supply, and $\dot{e}_{\xi}$ is the energy production due to energy exchange between constituents. The heat flux vector in the $\xi$-th constituent is $\mathbf{q}_{\xi}$. Here we see that $\mathbf{T}_{\xi}, p_{\xi}$, and $L_{\xi}$ each do work on the $\xi$-th constituent in different ways. Notice that because we have introduced the distention gradient $\mathbf{A}_{\xi}$, we see that the deformation gradient $\mathbf{F}_{\boldsymbol{\xi}}$ and the density $\bar{\rho}_{\xi}$ can do work independently through the terms containing the stress $\mathbf{T}_{\xi}$ and the pressure $p_{\xi}$. Because Hamilton's principle is based on energy considerations, Bedford and the author [6] were able to introduce these additional forces directly into the balance of momentum postulates.

From [6] the equation of conservation energy from the entire mixture is

$$
\sum_{\xi}\left(\dot{e}_{\xi}+\mathbf{b}_{\xi} \cdot \mathbf{v}_{\xi}\right)=0
$$

\section{$7 \quad$ Reduced System of Equations}

We next demonstrate that the equations of balance of momentum and conservation of energy from [6] are equivalent to those given in [8]. A major difference in these two formulations is the appearance of the Lagrange multipliers and the generalized forces $L_{\xi}$ in Eqs. (44) and (50). They do not appear in [8]. However, Eqs. (45) and (46) show how the Lagrange multipliers $\lambda$ and $\mu_{\xi}$ are connected to the pressures $p_{\xi}$, which do appear in [8]. We now show that the remaining terms $\tau$ and $L_{\xi}$ can be eliminated from our system of equations.

The first step in this process is to define a new momentum production term $\mathbf{m}_{\xi}^{+}$. It is

$$
\mathbf{m}_{\xi}^{+}=\mathbf{b}_{\xi}+c_{\xi}^{+}(\operatorname{grad} \tau-\mathbf{v}) .
$$


From Eqs. (32) and (49) the balance of momentum for the entire mixture now becomes

$$
\sum_{\xi} \mathbf{m}_{\xi}^{+}=0
$$

while the balance of momentum for the $\xi$-th constituent becomes

$$
\rho_{\xi} \mathbf{a}_{\xi}=\operatorname{div}\left(\mathbf{T}_{\xi}-\varphi_{\xi} \lambda \mathbf{1}\right)+\rho_{\xi} \mathbf{f}_{\xi}+\mathbf{m}_{\xi}^{+}+\lambda \operatorname{grad} \varphi_{\xi}-c_{\xi}^{+} \mathbf{u}_{\xi}
$$

Next we define a new energy production term $e_{\xi}^{+}$:

$$
e_{\xi}^{+}=\dot{e}_{\xi}-c_{\xi}^{+}\left(\frac{1}{2} \mathbf{u}_{\xi} \cdot \mathbf{u}_{\xi}+L_{\xi}-\frac{\lambda}{\bar{\rho}_{\xi}}\right)+\mathbf{m}_{\xi}^{+} \cdot \mathbf{v}_{\xi}
$$

From Eq. (51) the conservation of energy for the entire mixture becomes

$$
\sum_{\xi} e_{\xi}^{+}=0
$$

where Eq. (48) has been used. Then the conservation of energy for the $\xi$-th constituent becomes

$$
\begin{aligned}
\rho_{\xi} \dot{E}_{\xi}+ & c_{\xi}^{+} H_{\xi}=\operatorname{tr}\left(\mathbf{T}_{\xi}^{t} \mathcal{L}_{\xi}\right)+\varphi_{\xi} p_{\xi} \frac{\dot{\bar{\rho}}_{\xi}}{\bar{\rho}_{\xi}}-\operatorname{div} \mathbf{q}_{\xi}+\rho_{\xi} r_{\xi} \\
& +e_{\xi}^{+}-\mathbf{m}_{\xi}^{+} \cdot \mathbf{v}_{\xi}+\frac{1}{2} c_{\xi}^{+} \mathbf{u}_{\xi} \cdot \mathbf{u}_{\xi}
\end{aligned}
$$

where

$$
H_{\xi}=E_{\xi}+\frac{p_{\xi}}{\bar{\rho}_{\xi}} .
$$

At equilibrium, $H_{\xi}$ is the enthalpy of the $\xi$-th constituent. Thus $\tau$, $L_{\xi}$, and Eq. (48) have been eliminated from the mass, momentum, and energy postulates. After substitution of Eq. (37), the balance of energy becomes

$$
\begin{gathered}
\rho_{\xi} \dot{E}_{\xi}+c_{\xi}^{+} E_{\xi}=\operatorname{tr}\left[\left(\mathbf{T}_{\xi}^{t}-\varphi_{\xi} \lambda \mathbf{1}\right) \mathcal{L}_{\xi}\right]-\lambda \dot{\varphi}_{\xi} \\
-\operatorname{div} \mathbf{q}_{\xi}+\rho_{\xi} r_{\xi}+e_{\xi}^{+}-\mathbf{m}_{\xi}^{+} \cdot \mathbf{v}_{\xi}+\frac{1}{2} c_{\xi}^{+} \mathbf{u}_{\xi} \cdot \mathbf{u}_{\xi} .
\end{gathered}
$$

Equations (54) and (59) are equivalent to the equations of balance of momentum and conservation of energy in [8] when the terms associated with equilibrated forces and inertias are ignored. ${ }^{7}$

\footnotetext{
${ }^{7}$ The energy equations as postulated by Passman, Nunziato, and Walsh [8] involve an
} 


\section{Fluid-Saturated Porous Solid}

The kinematics and the equations of conservation of mass, balance of momentum, and conservation of energy have been reviewed in the preceding sections. To complete our theory we must prescribe a set of constitutive functions that describe the behavior of the constituents of our immiscible mixture. Baer and Nunziato [3] have used an immiscible mixture theory to develop a one-dimensional model for the burning and detonation of an explosive. Here we extend their work to three spatial dimensions; we use distention rather than volume fraction as a primitive constitutive variable; and we include elastic-plastic effects.

Consider an immiscible mixture of a solid $\xi=S$ and a fluid $\xi=G$. We assume that the solid forms a lattice of pores that contain a fluid. This means that the solid resists motion that changes the deformation gradient $\mathbf{F}_{S}$. This is the distinguishing feature of a porous solid. ${ }^{8}$ Recall that $\mathbf{T}_{S}$ is the conjugate variable for $\mathbf{F}_{S}$, while $p_{\xi}$ are the conjugate variables for $\bar{\rho}_{\xi}$. We shall also assume that the only way to do work on the fluid is by changing its true density. Thus

$$
\mathbf{T}_{G} \equiv \mathbf{0} \text {. }
$$

interesting manipulation of the energy production terms. Their original energy postulate does not explicitly contain a term with $p_{\xi}=\lambda$. However, they include such a term by introducing an "extra growth of energy" $e_{\xi}^{e}$ into the energy production $\dot{e}_{\xi}$ :

$$
e_{\xi}^{e} \equiv \lambda\left(\dot{\varphi}_{\xi}-\mathbf{v} \cdot \operatorname{grad} \varphi_{\xi}\right)
$$

While $e_{\xi}^{e}$ appears to be an ad-hoc definition it actually allows mixture constituents to do work on each other through the interface pressure $\lambda$ and the volume fraction constraint Eq. (3). Because of Eq. (51) the extra growth of energy also influences the balance of momentum where it produces the buoyancy term Eq. (10). Although this is a good motivation to include $e_{\xi}^{e}$, it takes an effect that is clearly kinematic and consigns it to a constitutive

assumption. One consequence of this approach is the need to include $\operatorname{grad} \varphi_{\xi}$ in the list of independent variables in the constitutive postulates. Baer and Nunziato [3] have overlooked this point in their derivation where they initially omit $\operatorname{grad} \varphi_{\xi}$ in their constitutive postulates but then include it later. \{Compare Eqs. (22) and (44) in [3].\} However, we point out that proper treatment of the kinematical constraint Eq. (3) eliminates the need for unnecessarily complicating the constitutive equations in this manner.

${ }^{8}$ In contrast to this we might consider solid particles suspended in a fluid. Ignoring viscous effects and assuming the particles do not touch, we see that it is possible to change $\mathbf{F}_{S}$ without doing work. 


\subsection{Reduced System of Equations}

Recall that we began our discussion of the equations of balance of momentum and conservation of energy by presenting the results of [6] and then by eliminating $\tau$ and $L_{\xi}$ we reduced these results to those of [8]. Here we must repeat this process again because by limiting the discussion to a binary mixture, Baer and Nunziato [3] were able to use an alternate set of reduced equations. The equations of conservation of mass come directly from Eq. (30):

$$
\begin{gathered}
\dot{\rho}_{S}+\rho_{S} \operatorname{div} \mathbf{v}_{S}=c_{S}^{+}, \\
\dot{\rho}_{G}+\rho_{G} \operatorname{div} \mathbf{v}_{G}=-c_{S}^{+}
\end{gathered}
$$

where from Eq. (32)

$$
c_{G}^{+}=-c_{S}^{+}
$$

Next instead of Eq. (52), we have

$$
\tilde{\mathbf{m}}_{\xi}^{+}=\mathbf{b}_{\xi}+c_{\xi}^{+}\left[\operatorname{grad} \tau-\frac{1}{2}\left(\mathbf{v}_{S}+\mathbf{v}_{G}\right)\right]
$$

where as in Section 7 it can be shown that

$$
\tilde{\mathbf{m}}_{G}^{+}=-\tilde{\mathbf{m}}_{S}^{+} \text {. }
$$

After Eq. (63) is substituted into Eq. (54), we obtain the following equations of balance of momentum for each constituent:

$$
\begin{aligned}
\rho_{S} \mathbf{a}_{S} & =\frac{1}{2} c_{S}^{+}\left(\mathbf{v}_{G}-\mathbf{v}_{S}\right)+\rho_{S} \mathbf{f}_{S}+\tilde{\mathbf{m}}_{S}^{+}-\varphi_{S} \operatorname{grad} \lambda+\operatorname{div} \mathbf{T}_{S}, \\
\rho_{G} \mathbf{a}_{G} & =\frac{1}{2} c_{S}^{+}\left(\mathbf{v}_{G}-\mathbf{v}_{S}\right)+\rho_{G} \mathbf{f}_{G}-\tilde{\mathbf{m}}_{S}^{+}-\varphi_{G} \operatorname{grad} \lambda .
\end{aligned}
$$

When Eqs. (48) and (63) are substituted into Eq. (51), we obtain

$$
\tilde{e}_{G}^{+}=-\tilde{e}_{S}^{+}
$$

where

$$
\tilde{e}_{\xi}^{+}=\dot{e}_{\xi}+\tilde{\mathbf{m}}_{\xi}^{+} \cdot \mathbf{v}_{\xi}-c_{\xi}^{+}\left(L_{\xi}-\frac{\lambda}{\bar{\rho}_{\xi}}\right) .
$$

The conservation of energy for each constituent Eq. (50) becomes

$$
\begin{aligned}
\rho_{S} \dot{E}_{S}+c_{S}^{+} H_{S}= & \operatorname{tr}\left(\mathbf{T}_{S}^{t} \mathcal{L}_{S}\right)+\varphi_{S} p_{S} \frac{\dot{\bar{\rho}}_{S}}{\bar{\rho}_{S}}+\tilde{e}_{S}^{+}-\tilde{\mathbf{m}}_{S}^{+} \cdot \mathbf{v}_{S} \\
& -\operatorname{div} \mathbf{q}_{S}+\rho_{S} r_{S}, \\
\rho_{G} \dot{E}_{G}-c_{S}^{+} H_{G}= & \varphi_{G} p_{G} \frac{\dot{\bar{\rho}}_{G}}{\bar{\rho}_{G}}-\tilde{e}_{S}^{+}+\tilde{\mathbf{m}}_{S}^{+} \cdot \mathbf{v}_{G}-\operatorname{div} \mathbf{q}_{G}+\rho_{G} r_{G} .
\end{aligned}
$$


Later we shall show how these balance laws can be combined with specific constitutive assumptions to yield the equations of Baer and Nunziato [3].

\subsection{Constitutive Assumptions}

We now introduce three new quantities into our theory. They are the Helmholtz free energy $\psi_{\xi}$, the entropy $\eta_{\xi}$, and the temperature $\vartheta_{\xi}$. They are related to the internal energy by the Legendre transformation

$$
\psi_{\xi}=E_{\xi}-\vartheta_{\xi} \eta_{\xi}
$$

It will also be convenient to define the temperature gradient $\mathbf{G}_{\xi}$, where

$$
\mathbf{G}_{\xi} \equiv \operatorname{grad} \vartheta_{\xi}
$$

Now we decompose the distention and the true deformation gradient of the solid into elastic $E$ and plastic $P$ parts as follows:

$$
\mathbf{A}_{S}=\mathbf{A}_{S}^{E} \mathbf{A}_{S}^{P}
$$

and

$$
\overline{\mathbf{F}}_{S}=\overline{\mathbf{F}}_{S}^{E} \overline{\mathbf{F}}_{S}^{P}
$$

We make the usual assumption that the plastic deformation is incompressible

$$
\operatorname{det} \overline{\mathbf{F}}_{S}^{P} \equiv 1
$$

where a similar assumption does not hold for the plastic distention gradient. From the true conservation of mass Eq. (35) we find that

$$
\operatorname{det} \overline{\mathbf{F}}_{S}^{E}=\frac{\bar{\rho}_{S 0}}{\bar{\rho}_{S}} \text {. }
$$

Next we define three sets of primitive constitutive variables:

$$
\begin{aligned}
\Lambda_{S} & \equiv\left\{\overline{\mathbf{F}}_{S}^{E}, \mathbf{A}_{S}^{E}, \vartheta_{S}, \dot{\overline{\mathbf{F}}}_{S}^{E}, \dot{\mathbf{A}}{ }_{S}^{E}, \mathbf{G}_{S}, d_{S}\right\} \\
\Lambda_{G} & \equiv\left\{\bar{\rho}_{G}, \vartheta_{G}, \mathbf{G}_{G}\right\} \\
\Lambda_{M} & \equiv\left\{\Lambda_{S}, \Lambda_{G}, \mathbf{v}_{F}, \mathbf{v}_{G}\right\}
\end{aligned}
$$

The variable $d_{S}$ is a scalar that represents damage to the solid matrix in the form of cracks that do not necessarily contribute to the 
distention of the solid. The primitive constitutive variables contained in $\Lambda_{S}$ describe the state of the solid, those in $\Lambda_{G}$ describe the state of the fluid, and those in $\Lambda_{M}$ describe the state of the mixture. Notice that only the elastic portions of the true deformation gradient and the distention gradient appear in $\Lambda_{S}$. These primitive variables are the independent variables in the constitutive equations of the mixture. The set of constitutive functions $f_{S}$ for the solid are

$$
\left\{\psi_{S}, \eta_{S}, \mathbf{T}_{S}, p_{S}, \mathbf{q} \dot{\mathbf{F}}_{S}^{P}, \dot{\mathbf{A}}_{S}^{P}, \dot{d}_{S}\right\}=f_{S}\left(\Lambda_{S}\right)
$$

Notice that while $\overline{\mathbf{F}}_{S}^{E}$ and $\mathbf{A}_{S}^{E}$ are primitive variables, we write constitutive relations for their counterparts $\dot{\overline{\mathbf{F}}}_{S}^{P}$ and $\dot{\mathrm{A}}_{S}^{P}$. These types of relationships are often called plastic flow rules. To complete the constitutive assumptions we assume that the constitutive relations for the fluid are

$$
\left\{\psi_{G}, \eta_{G}, p_{G}, \mathbf{q}_{G}\right\}=f_{G}\left(\Lambda_{G}\right)
$$

and those for the mixture are

$$
\left\{\tilde{e}_{S}^{+}, \mathbf{m}_{S}^{+}, c_{S}^{+}\right\}=f_{M}\left(\Lambda_{M}\right)
$$

These constitutive relations reflect the application of what we call the immiscibility postulate, also called the principle of phase separation. This postulate requires that the constitutive equations of the $\xi$-th constituent only depend upon the primitive variables $\Lambda_{\xi}$, while the mixture production terms depend upon all of the primitive variables.

\subsection{Admissible Thermodynamic Processes}

With the statement of the constitutive assumptions, we have a theory in which the number of unknown variables equals the number of equations. We have written the constitutive assumptions in a manner that suggests that we are free to assume any functional form containing the primitive variables. This is not true, and in fact many constraints apply that limit the allowed functional forms. In order to investigate the nature of these constraints, it is convenient to define an admissible thermodynamic process. This is a solution that satisfies the field equations of the theory without regard to the boundary conditions. The set of all such solutions is useful for investigating the constitutive constraints imposed by the second law of thermodynamics (see 
[12]). The following discussion also serves as a summary of the field equations of the theory.

- Suppose that we know the solutions for the motion and the temperature of each constituent

$$
\begin{aligned}
\mathbf{x} & =\chi_{\xi}\left(\mathbf{X}_{\xi}, t\right), \\
\vartheta_{\xi} & =\vartheta_{\xi}\left(\mathbf{X}_{\xi}, t\right) .
\end{aligned}
$$

We now show how to satisfy all of the field equations for the mixture given these solutions. Substitution of these solutions into Eqs. (12), (13), (16), and (70) yield solutions for $\mathbf{v}_{\xi}, \mathbf{a}_{\xi}, \mathbf{F}_{\xi}$, and $\mathbf{G}_{\xi}$. Next we consider a set of 21 equations. They are Eqs. (3), (6), (17), (21), (35), $(47),(61),(71)$, and $(72)$, plus the constitutive equations for $p_{\xi}, \dot{\overline{\mathbf{F}}}_{S}^{P}$, $\dot{\mathrm{A}}_{S}^{P}, \dot{d}_{S}$, and $c_{S}^{+}$from Eqs. (76), (77), and (78). We assume these equations can be integrated to obtain solutions for the unknowns in this set, $\mathbf{A}_{\xi}, \mathbf{A}_{S}^{E}, \mathbf{A}_{S}^{P}, \overline{\mathbf{F}}_{\xi}, \overline{\mathbf{F}}_{S}^{E}, \overline{\mathbf{F}}_{S}^{P}, \bar{\rho}_{\xi}, \bar{J}_{\xi}, \rho_{\xi}, c_{S}^{+}, \varphi_{\xi}, d_{S}, \lambda$, and $p_{\xi}$. The remaining constitutive relations can then be evaluated to obtain $\psi_{\xi}, \eta_{\xi}, \mathbf{q}_{\xi}, \mathbf{T}_{S}, \tilde{\mathbf{m}}_{S}^{+}$, and $e_{S}^{+}$. The internal energies $E_{\xi}$ are then determined by Eqs. (69). Finally we select a set of external body forces $\mathbf{b}_{\xi}$ and heat supplies $r_{\xi}$ that satisfy the balance of momentum Eq. (65) and conservation of energy Eq. (68).

\subsection{Second Law of Thermodynamics}

The constitutive relations must obey the constraints imposed by the second law of thermodynamics through the following dissipation inequality:

$$
\sum_{\xi}\left[\rho_{\xi} \dot{\eta}_{\xi}+\operatorname{div}\left(\frac{\mathbf{q}_{\xi}}{\vartheta_{\xi}}\right)-\frac{\rho_{\xi} r_{\xi}}{\vartheta_{\xi}}+c_{\xi}^{+} \eta_{\xi}\right] \geq 0 .
$$

Coleman and Noll originally argued that the constitutive relationships must be written in such a way as to simultaneously satisfy Eq. (79) for all possible admissible thermodynamic processes (see [12], page 250). We apply their arguments here by first solving the conservation of energy Eqs. (68) for $\rho_{\xi} r_{\xi}$ and substituting the result into this inequality. Then the transformations Eq. (69) are applied, and the derivatives of 
the $\psi_{\xi}$ are expanded using the chain rule. The result is

$$
\begin{aligned}
& -\frac{\rho_{S}}{\vartheta_{S}}\left\{\left(\eta_{S}+\frac{\partial \psi_{S}}{\partial \vartheta_{S}}\right) \dot{\vartheta}_{S}+\operatorname{tr}\left[\left(\frac{\partial \psi_{S}}{\partial \dot{\overline{\mathbf{F}}}}\right)^{t} \ddot{\overline{\mathbf{F}}}_{S}^{E}+\left(\frac{\partial \psi_{S}}{\partial \dot{\mathbf{A}}_{S}^{E}}\right)^{t} \ddot{\mathbf{A}}_{S}^{E}\right.\right. \\
& \left.\left.+\left(\frac{\partial \psi_{S}}{\partial \mathbf{G}_{S}}\right)^{t} \dot{\mathbf{G}}_{S}+\left(\frac{\partial \psi_{S}}{\partial \overline{\mathbf{F}}_{S}^{E}}\right)^{t} \dot{\overline{\mathbf{F}}}_{S}^{E}+\left(\frac{\partial \psi_{S}}{\partial \mathbf{A}_{S}^{E}}\right)^{t} \dot{\mathbf{A}}_{S}^{E}\right]+\frac{\partial \psi_{S}}{\partial d_{S}} \dot{d}_{S}\right\} \\
& +\frac{\operatorname{tr}\left(\mathbf{T}_{S}^{t} \mathcal{L}_{S}\right)}{\vartheta_{S}}+\frac{\varphi_{S} p_{S}}{\vartheta_{S}} \frac{\dot{\bar{\rho}}_{S}}{\bar{\rho}_{S}} \\
& -\frac{\rho_{G}}{\vartheta_{G}}\left\{\operatorname{tr}\left[\left(\frac{\partial \psi_{G}}{\partial \mathbf{G}_{G}}\right)^{t} \dot{\mathbf{G}}_{G}\right]+\left(\eta_{G}+\frac{\partial \psi_{G}}{\partial \vartheta_{G}}\right) \dot{\vartheta}_{G}\right\} \\
& +\frac{\varphi_{G}}{\vartheta_{G}}\left(p_{G}-\bar{\rho}_{G}^{2} \frac{\partial \psi_{G}}{\partial \bar{\rho}_{G}}\right) \frac{\dot{\bar{\rho}}_{G}}{\bar{\rho}_{G}}-c_{S}^{+}\left(\frac{\mathcal{G}_{S}}{\vartheta_{S}}-\frac{\mathcal{G}_{G}}{\vartheta_{G}}\right)-\tilde{\mathbf{m}}_{S}^{+} \cdot\left(\frac{\mathbf{v}_{S}}{\vartheta_{S}}-\frac{\mathbf{v}_{G}}{\vartheta_{G}}\right) \\
& -\frac{\mathbf{q}_{S} \cdot \mathbf{G}_{S}}{\vartheta_{S}^{2}}-\frac{\mathbf{q}_{G} \cdot \mathbf{G}_{G}}{\vartheta_{G}^{2}}+\tilde{e}_{S}^{+}\left(\frac{1}{\vartheta_{S}}-\frac{1}{\vartheta_{G}}\right) \geq 0
\end{aligned}
$$

where ()$^{t}$ represents the transpose of the argument and where we have defined

$$
\mathcal{G}_{\xi}=H_{\xi}-\vartheta_{\xi} \eta_{\xi}
$$

At equilibrium $\mathcal{G}_{\xi}$ is the Gibb's energy. This inequality must be satisfied for all admissible thermodynamic processes. Notice that a set of admissible processes exists in which $\dot{\vartheta}_{\xi}, \ddot{\mathbf{F}}_{S}^{E}, \ddot{\mathbf{A}}_{S}^{E}, \dot{\mathbf{G}}_{\xi}$, and $\dot{\bar{\rho}}_{G}$ assume arbitrarily large positive and negative values while all other quantities in the inequality are left unchanged. If the inequality is to be satisfied by all the solutions in this set, the coefficients of $\dot{\vartheta}_{\xi}, \ddot{\mathbf{F}}_{S}^{E}, \ddot{\mathbf{A}}_{S}^{E}, \dot{\mathbf{G}}_{\xi}$, and $\dot{\bar{\rho}}_{G}$ must be zero. This means that $\psi_{S}$ cannot be a function of $\dot{\mathbf{F}}_{S}$, $\dot{\mathbf{A}} \dot{E}_{S}$, or $\mathbf{G}_{S}$, and therefore

$$
\psi_{S}=\psi_{S}\left(\overline{\mathbf{F}}_{S}^{E}, \vartheta_{S}, \mathbf{A}_{S}^{E}, d_{S}\right)
$$

Similarly

$$
\begin{gathered}
\psi_{G}=\psi_{G}\left(\bar{\rho}_{G}, \vartheta_{G}\right), \\
\eta_{\xi}=-\frac{\partial \psi_{\xi}}{\partial \vartheta_{\xi}}
\end{gathered}
$$

and

$$
p_{G}=\bar{\rho}_{G}^{2} \frac{\partial \psi_{G}}{\partial \bar{\rho}_{G}}
$$


Thus all of the constitutive equations for the fluid with the exception of $\mathbf{q}_{G}$ are determined by the Helmholtz energy $\psi_{G}$.

Next we notice that

$$
\begin{gathered}
\operatorname{tr}\left(\mathbf{T}_{S}^{t} \mathcal{L}_{S}\right)=\operatorname{tr}\left[\mathbf{T}_{S}^{t} \dot{\mathbf{A}}_{S}^{E}\left(\mathbf{A}_{S}^{E}\right)^{-1}\right]+\operatorname{tr}\left[\left(\mathbf{A}_{S}^{E}\right)^{-1} \mathbf{T}_{S}^{t} \mathbf{A}_{S}^{E} \dot{\mathbf{A}}_{S}^{P}\left(\mathbf{A}_{S}^{P}\right)^{-1}\right] \\
\quad \tilde{+} \operatorname{tr}\left[\mathbf{A}_{S}^{-1} \mathbf{T}_{S}^{t} \mathbf{A}_{S} \dot{\mathbf{F}_{S}}\left(\overline{\mathbf{F}}_{S}^{E}\right)^{-1}\right]+\operatorname{tr}\left[\mathbf{F}_{S}^{-1} \mathbf{T}_{S}^{t} \mathbf{F}_{S}\left(\overline{\mathbf{F}}_{S}^{P}\right)^{-1} \dot{\overline{\mathbf{F}}}_{S}^{P}\right],
\end{gathered}
$$

and from Eq. (74),

$$
\frac{\dot{\bar{\rho}}_{S}}{\bar{\rho}_{S}}=-\operatorname{tr}\left[\dot{\overline{\mathbf{F}}}_{S}^{E}\left(\overline{\mathbf{F}}_{S}^{E}\right)^{-1}\right] .
$$

When these expressions are substituted into the inequality (80), we obtain

$$
\begin{aligned}
& \frac{1}{\vartheta_{S}}\left\{\operatorname{tr}\left[\left(\mathbf{T}_{S}^{t}-\rho_{S} \mathbf{A}_{S}^{E}\left(\frac{\partial \psi_{S}}{\partial \mathbf{A}_{S}^{E}}\right)^{t}\right) \dot{\mathbf{A}}_{S}^{E}\left(\mathbf{A}_{S}^{E}\right)^{-1}\right]\right. \\
& +\operatorname{tr}\left[\left(\mathbf{A}_{S}^{E}\right)^{-1} \mathbf{T}_{S}^{t} \mathbf{A}_{S}^{E} \dot{\mathbf{A}}_{S}^{P}\left(\mathbf{A}_{S}^{P}\right)^{-1}\right] \\
& +\operatorname{tr}\left[\left(\mathbf{A}_{S}^{-1} \mathbf{T}_{S}^{t} \mathbf{A}_{S}-\rho_{S} \overline{\mathbf{F}}_{S}^{E}\left(\frac{\partial \psi_{S}}{\partial \overline{\mathbf{F}}_{S}^{E}}\right)^{t}-\varphi_{S} p_{S} \mathbf{1}\right) \dot{\overline{\mathbf{F}}}_{S}^{E}\left(\overline{\mathbf{F}}_{S}^{E}\right)^{-1}\right] \\
& \left.+\operatorname{tr}\left[\mathbf{F}_{S}^{-1} \mathbf{T}_{S}^{t} \mathbf{F}_{S}\left(\overline{\mathbf{F}}_{S}^{P}\right)^{-1} \dot{\mathbf{F}}_{S}^{P}\right]-\rho_{S} \frac{\partial \psi_{S}}{\partial d_{S}} \dot{d}_{S}\right\}-\frac{\mathbf{q}_{S} \cdot \mathbf{G}_{S}}{\vartheta_{S}^{2}}-\frac{\mathbf{q}_{G} \cdot \mathbf{G}_{G}}{\vartheta_{G}^{2}} \\
& -c_{S}^{+}\left(\frac{\mathcal{G}_{S}}{\vartheta_{S}}-\frac{\mathcal{G}_{G}}{\vartheta_{G}}\right)-\tilde{\mathbf{m}}_{S}^{+} \cdot\left(\frac{\mathbf{v}_{S}}{\vartheta_{S}}-\frac{\mathbf{v}_{G}}{\vartheta_{G}}\right)+\tilde{e}_{S}^{+}\left(\frac{1}{\vartheta_{S}}-\frac{1}{\vartheta_{G}}\right) \geq 0
\end{aligned}
$$

Even after we satisfy Eqs. (82)-(85), our constitutive postulates must also satisfy this dissipation inequality. We notice that the inequality is composed of the sum of several terms. The most general constitutive models need only satisfy the inequality for this sum. However, we can also construct more specialized constitutive models that satisfy the inequality term by term. This is the method we shall adopt here. Thus we shall postulate special constitutive equations that are sufficient but not always necessary to satisfy the inequality.

\subsection{Reference Solid}

The reference solid is a useful concept in the theory of porous solids [12]. Suppose for a moment that our solid is undamaged and undistended so that the mixture degenerates to a single nonporous solid 
that we shall call the reference solid. Then by definition $\mathbf{A}_{S}=1$ and $d_{S}=0$. The Helmholtz energy of this material is

$$
\psi_{S}=\psi_{S R}\left(\overline{\mathbf{F}}_{S}^{E}, \vartheta_{S}\right) \equiv \psi_{S}\left(\overline{\mathbf{F}}_{S}^{E}, \vartheta_{S}, \mathbf{1}, 0\right)
$$

The function $\psi_{S R}\left(\overline{\mathbf{F}}_{S}^{E}, \vartheta_{S}\right)$ is the Helmholtz energy of the reference solid. The equilibrium stress $\overline{\mathbf{T}}_{R e}$ of the reference solid is defined to be

$$
\overline{\mathbf{T}}_{R e} \equiv \rho_{S} \frac{\partial \psi_{S R}}{\partial \overline{\mathbf{F}}_{S}^{E}}\left(\overline{\mathbf{F}}_{S}^{E}\right)^{t},
$$

and equilibrium true stress $\overline{\mathbf{T}}_{S e}$ of the mixture is defined to be

$$
\overline{\mathbf{T}}_{S e} \equiv \rho_{S} \frac{\partial \psi_{S}}{\partial \overline{\mathbf{F}}_{S}^{E}}\left(\overline{\mathbf{F}}_{S}^{E}\right)^{t}
$$

Thus

$$
\overline{\mathbf{T}}_{R e}=\overline{\mathbf{T}}_{S e}\left(\overline{\mathbf{F}}_{S}^{E}, \vartheta_{S}, \mathbf{1}, 0\right) .
$$

In some cases it may be reasonable to assume that

$$
\psi_{S}=\psi_{S R}\left(\overline{\mathbf{F}}_{S}^{E}, \vartheta_{S}\right)+\psi_{S A}\left(\vartheta_{S}, \mathbf{A}_{S}^{E}, d_{S}\right)
$$

In such cases we see that

$$
\overline{\mathbf{T}}_{S e}=\overline{\mathbf{T}}_{R e}
$$

for all values of the distention gradient $\mathbf{A}_{S}$ and the damage $d_{S}$.

\subsection{Equilibrium States}

The dissipation inequality ( 88 ) must be zero at equilibrium. We define equilibrium to be states where

$$
\begin{gathered}
\dot{\mathbf{A}}_{S}^{E}=\dot{\mathbf{A}}_{S}^{P}=\mathbf{0}, \quad \dot{\mathbf{F}}_{S}=\dot{\mathbf{F}}_{S}^{P}=\mathbf{0}, \\
\dot{d}_{S}=0, \quad \mathbf{q}_{\xi}=\mathbf{0}, \quad c_{S}^{+}=0, \quad \tilde{\mathbf{m}}_{S}^{+}=\mathbf{0}, \quad \tilde{e}_{S}^{+}=0 .
\end{gathered}
$$

About every equilibrium state we assume that regions exist in which the solid constituent is elastic and neither damage nor plastic deformation occur. Therefore we must require that the constitutive relationships for the quantities $\dot{\overline{\mathbf{F}}}_{S}^{P}, \dot{\mathbf{A}}_{S}^{P}$, and $\dot{d}_{S}$ are zero in these neighborhoods of the equilibrium state. This assumption of elastic 
behavior also implies that the coefficients of the terms $\dot{\mathbf{A}}_{S}^{E}\left(\mathbf{A}_{S}^{E}\right)^{-1}$ and $\dot{\overline{\mathbf{F}}}_{S}^{E}\left(\overline{\mathbf{F}}_{S}^{E}\right)^{-1}$ in Eq. (88) are finite and nonzero at arbitrarily small distances from the equilibrium states. In order for the inequality to be at its minimum value at equilibrium, the coefficients of $\dot{\mathbf{A}}_{S}^{E}\left(\mathbf{A}_{S}^{E}\right)^{-1}$ and $\dot{\overline{\mathbf{F}}}_{S}^{E}\left(\overline{\mathbf{F}}_{S}^{E}\right)^{-1}$ must be zero at equilibrium. Thus

$$
\left.\begin{array}{rl}
\mathbf{T}_{S} & =\mathbf{T}_{S e} \\
p_{S} & =p_{S e} \\
\mathbf{T}_{S e}^{t} & =\mathbf{A}_{S} \overline{\mathbf{T}}_{S e}^{t} \mathbf{A}_{S}^{-1}+\varphi_{S} p_{S e} \mathbf{1}
\end{array}\right\} \text { at equilibrium }
$$

where the equilibrium stress $\mathbf{T}_{S e}$ is

$$
\mathbf{T}_{S e} \equiv \rho_{S} \frac{\partial \psi_{S}}{\partial \mathbf{A}_{S}^{E}}\left(\mathbf{A}_{S}^{E}\right)^{t}
$$

Hence the equilibrium pressure $p_{S e}$ is

$$
\varphi_{S} p_{S e} \equiv \frac{1}{3} \operatorname{tr}\left(\mathbf{T}_{S e}-\overline{\mathbf{T}}_{S e}\right) .
$$

Now we split the stress $\mathbf{T}_{S}$ into two parts

$$
\mathbf{T}_{S}=-\varphi_{S} \beta_{S} \mathbf{1}+\mathbf{T}_{S}^{\prime}
$$

where the pressure $\beta_{S}$ and the equilibrium pressure $\beta_{S e}$ are

$$
-\varphi_{S} \beta_{S} \equiv \frac{1}{3} \operatorname{tr} \mathbf{T}_{S}, \quad-\varphi_{S} \beta_{S e} \equiv \frac{1}{3} \operatorname{tr} \mathbf{T}_{S e} .
$$

The deviatoric stress $\mathbf{T}_{S}^{\prime}$ obeys

$$
\operatorname{tr}\left(\mathbf{T}_{S}^{\prime}\right)=0
$$

Then Eq. (98) yields

$$
p_{S e}=\bar{p}_{S e}-\beta_{S e}
$$

where

$$
\varphi_{S} \bar{p}_{S e} \equiv-\frac{1}{3} \operatorname{tr} \overline{\mathbf{T}}_{S e} .
$$

When these results are combined with Eqs. (47), we obtain ${ }^{9}$

$$
\bar{p}_{S e}-\beta_{S e}=p_{G} \text { at equilibrium. }
$$

\footnotetext{
${ }^{9}$ Here you should notice some subtle differences in nomenclature. For example our interface pressure $\lambda$ is called $\pi$ by Passman, Nunziato, and Walsh [8]; that is,

$$
\lambda=\tilde{\pi}
$$
}


Because of Eq. (85), $p_{G}$ is always an equilibrium pressure.

Similar arguments show that at equilibrium, the coefficients of $\mathbf{q}_{\xi}$, $c_{S}^{+}, \tilde{\mathbf{m}}_{S}^{+}$, and $e_{S}^{+}$must also be zero, and therefore

$\mathbf{G}_{\xi}=\mathbf{0}, \quad \mathcal{G}_{S}=\mathcal{G}_{G}, \quad \mathbf{v}_{S}=\mathbf{v}_{G}$, and $\vartheta_{S}=\vartheta_{G}$ at equilibrium. (105)

Because $\dot{\overline{\mathrm{F}}}_{S}^{P}, \dot{\mathrm{A}}_{S}^{P}$, and $\dot{d}_{S}$ are zero in some finite neighborhood of equilibrium, the coefficients of the terms $\left(\overline{\mathbf{F}}_{S}^{P}\right)^{-1} \dot{\overline{\mathbf{F}}}_{S}^{P}, \dot{\mathbf{A}}_{S}^{P}\left(\mathbf{A}_{S}^{P}\right)^{-1}$, and $\dot{d}_{S}$ in Eq. (88) are not required to be zero at the equilibrium states.

\subsection{Flow Rules}

We shall choose constitutive assumptions that are sufficient to satisfy the dissipation inequality Eq. (88). We do this by satisfying the inequality term by term. Two such constraints on our constitutive postulates are:

$$
\begin{gathered}
\operatorname{tr}\left[\left(\mathbf{A}_{S}^{E}\right)^{-1} \mathbf{T}_{S}^{t} \mathbf{A}_{S}^{E} \dot{\mathbf{A}}_{S}^{P}\left(\mathbf{A}_{S}^{P}\right)^{-1}\right] \geq 0 \\
\operatorname{tr}\left(\mathbf{F}_{S}^{-1} \mathbf{T}_{S}^{t} \mathbf{F}_{S}\left(\overline{\mathbf{F}}_{S}^{P}\right)^{-1} \dot{\mathbf{F}}_{S}^{P}\right) \geq 0
\end{gathered}
$$

Here we shall postulate flow rules for $\dot{\mathbf{A}}_{S}^{P}$ and $\dot{\overline{\mathbf{F}}}_{S}^{P}$ that satisfy these individual inequalities. First notice that $\dot{\overline{\mathrm{F}}}_{S}$ is already constrained by Eq. (73). Taking the material derivative of this expression yields

$$
\operatorname{tr}\left[\left(\overline{\mathbf{F}}_{S}^{P}\right)^{-1} \dot{\mathbf{F}}_{S}^{P}\right]=0 .
$$

where we have added the tilde over the $\pi$ to denote that it is the nomenclature of [8]. Moreover in [8], $\tilde{\lambda}_{\xi}$ represents "microstructural viscosity." Now notice that when we ignore the equilibrated stress and body force terms in Eq. (5C.7.7) of [8] then at equilibrium $\tilde{\pi}=\tilde{p}_{\xi}-\tilde{\beta}_{\xi}$. Thus from Eq. (46) we see another important distinction in nomenclature; that is, at equilibrium

$$
p_{\xi}=\tilde{p}_{\xi}-\tilde{\beta}_{\xi} .
$$

This equation illustrates how our pressure $p_{\xi}$ is related to the constituent pressure $\tilde{p}_{\xi}$ and configurational stress $\tilde{\beta}_{\xi}$ in [8]. This difference in nomenclature arises because we assume that the set $\left(p_{\xi}, \bar{\rho}_{\xi}\right)$ is a conjugate pair and $p_{\xi}$ does work when $\bar{\rho}_{\xi}$ changes. Whereas, Passman, Nunziato, and Walsh assume that $\left(\tilde{p}_{\xi}, \varphi_{\xi}\right)$ are a conjugate pair and $\tilde{p}_{\xi}$ does work as $\varphi_{\xi}$ changes. 
We account for this additional constraint on the values of $\overline{\mathbf{F}}_{S}^{P}$ by combining Eqs. (99), (106), and (107) to obtain

$$
\operatorname{tr}\left[\mathbf{F}_{S}^{-1}\left(\mathbf{T}_{S}^{\prime}\right)^{t} \mathbf{F}_{S}\left(\overline{\mathbf{F}}_{S}^{P}\right)^{-1} \dot{\mathbf{F}}_{S}^{P}\right] \geq 0
$$

We can now satisfy the inequalities with the following flow rules:

$$
\begin{array}{r}
\dot{\mathbf{A}}_{S}^{P}\left(\mathbf{A}_{S}^{P}\right)^{-1}=Y^{A}\left(\Lambda_{S}\right) \mathbf{S}^{A} \\
\left(\overline{\mathbf{F}}_{S}^{P}\right)^{-1} \dot{\mathbf{F}}_{S}^{P}=Y^{F}\left(\Lambda_{S}\right) \mathbf{S}^{F}
\end{array}
$$

where

$$
\begin{gathered}
\mathbf{S}^{A}=\left(\mathbf{A}_{S}^{E}\right)^{-1} \mathbf{T}_{S} \mathbf{A}_{S}^{E}, \\
\mathbf{S}^{F}=\mathbf{F}_{S}^{-1} \mathbf{T}_{S}^{\prime} \mathbf{F}_{S}
\end{gathered}
$$

and $Y^{A}$ and $Y^{F}$ are positive scalar constitutive functions of the set of primitive variables $\Lambda_{S}$. Notice that the flow rule for $\dot{\overline{\mathrm{F}}}_{S}$ satisfies Eq. (107). Recall that we must specify functions for $Y^{A}$ and $Y^{F}$ that are zero in a neighborhood of the equilibrium states. The boundaries of these neighborhoods are called yield surfaces.

\subsection{Damage}

A constraint on the constitutive postulate for damage that is sufficient to satisfy the damage term in Eq. (88) is

$$
-\frac{\partial \psi_{S}}{\partial d_{S}} \dot{d}_{S} \geq 0
$$

To satisfy this inequality we specify the following evolution equation for damage:

$$
\dot{d}_{S}=\mathcal{D}_{S}\left(S_{D}\right)
$$

where the sign of the function $\mathcal{D}_{S}$ must be equal to the sign of its argument $S_{D}$,

$$
S_{D}=-\frac{\partial \psi_{S}}{\partial d_{S}}
$$

Recall that this constitutive relationship must have a neighborhood about equilibrium where $\dot{d}_{S}=0$. The boundary of this specified neighborhood is called the threshold of damage. 


\subsection{Symmetry of the Distention Gradient}

From Eqs. (47) and (96) the equilibrium condition for the stress of a porous solid without pore pressure, $p_{G}=0$, is

$$
\mathbf{T}_{S e}^{t}=\mathbf{A}_{S} \overline{\mathbf{T}}_{S e}^{t} \mathbf{A}_{S}^{-1} \text {. }
$$

Often it is reasonable to assume that [see Eq. (94)]

$$
\mathbf{T}_{S e}^{t}=\mathbf{A}_{S} \overline{\mathbf{T}}_{R e}^{t} \mathbf{A}_{S}^{-1} \text {. }
$$

Now the stress must be symmetric in both the distended solid and the reference solid. It also seems reasonable to require symmetry of the true equilibrium stress $\overline{\mathbf{T}}_{S e}$. Thus $\mathbf{A}_{S}$ must transform a symmetric tensor $\overline{\mathbf{T}}_{S e}$ to another symmetric tensor $\mathbf{T}_{S e}$. Through the polar decomposition of $\mathbf{A}_{S}$ we find that it will have this transformation property if

$$
\mathbf{A}_{S}=\left(\alpha_{S}\right)^{\frac{1}{3}} \mathbf{R}_{S}
$$

where $\alpha_{S}$ is defined by Eq. (22) and $\mathbf{R}_{S}$ is a rotation tensor, which has the properties

$$
\left(\mathbf{R}_{S}\right)^{-1}=\mathbf{R}_{S}^{t}, \quad \operatorname{det} \mathbf{R}_{S}=1
$$

Thus the distention gradient can at most change the scalar distention $\alpha_{S}$ and cause a simple rotation of the material. The source of this limitation on $\mathbf{A}_{S}$ can be traced to our assumption that $p_{S}$ is a scalar. In the primary references this assumption was used to define force and work terms in the balance of momentum and conservation of energy.

\subsection{Distention and Pore Collapse}

In general, the distention gradient $\mathbf{A}_{S}$ is a second order tensor. We have found that $\mathbf{A}_{S}$ must be of the form shown in $\mathrm{Eq}$. (118). Suppose we assume that $R=1$ so that

$$
\mathbf{A}_{S}=\left(\alpha_{S}\right)^{\frac{1}{3}} 1, \quad \mathbf{A}_{S}^{P}=\left(\alpha_{S}^{P}\right)^{\frac{1}{3}} 1, \quad \mathbf{A}_{S}^{E}=\left(\alpha_{S}^{E}\right)^{\frac{1}{3}} 1 .
$$

Then the dissipation inequality $\mathrm{Eq}$. (88) becomes 


$$
\begin{aligned}
& \frac{1}{\vartheta_{S}}\left\{-\varphi_{S}\left(\beta_{S}+3 \bar{\rho}_{S} \alpha_{S}^{E} \frac{\partial \psi_{S}}{\partial \alpha_{S}^{E}}\right) \frac{\dot{\alpha}_{S}^{E}}{\alpha_{S}^{E}}-\varphi_{S} \beta_{S} \frac{\dot{\alpha}_{S}^{P}}{\alpha_{S}^{P}}\right. \\
& +\operatorname{tr}\left[\left(\mathbf{T}_{S}^{t}-\overline{\mathbf{T}}_{S e}^{t}-\varphi_{S} p_{S} \mathbf{1}\right) \dot{\overline{\mathbf{F}}}_{S}^{E}\left(\overline{\mathbf{F}}_{S}^{E}\right)^{-1}\right] \\
& \left.+\operatorname{tr}\left[\mathbf{F}_{S}^{-1} \mathbf{T}_{S}^{t} \mathbf{F}{ }_{S}\left(\overline{\mathbf{F}}_{S}^{P}\right)^{-1} \dot{\overline{\mathbf{F}}}_{S}^{P}\right]-\rho_{S} \frac{\partial \psi_{S}}{\partial d_{S}} \dot{d}_{S}\right\}-\frac{\mathbf{q}_{S} \cdot \mathbf{G}_{S}}{\vartheta_{S}^{2}}-\frac{\mathbf{q}_{G} \cdot \mathbf{G}_{G}}{\vartheta_{G}^{2}} \\
& -c_{S}^{+}\left(\frac{\mathcal{G}_{S}}{\vartheta_{S}}-\frac{\mathcal{G}_{G}}{\vartheta_{G}}\right)-\tilde{\mathbf{m}}_{S}^{+} \cdot\left(\frac{\mathbf{v}_{S}}{\vartheta_{S}}-\frac{\mathbf{v}_{G}}{\vartheta_{G}}\right)+\tilde{e}_{S}^{+}\left(\frac{1}{\vartheta_{S}}-\frac{1}{\vartheta_{G}}\right) \geq 0 .
\end{aligned}
$$

The equilibrium conditions and the flow rules for the mixture are unchanged except we see that now

$$
\beta_{S e}=-3 \bar{\rho}_{S} \alpha_{S}^{E} \frac{\partial \psi_{S}}{\partial \alpha_{S}^{E}}
$$

The following inequalities are sufficient to satisfy the first three terms of Eq. (121):

$$
\begin{aligned}
-\left(\beta_{S}-\beta_{S e}\right) \frac{\dot{\alpha}_{S}^{E}}{\alpha_{S}^{E}} & \geq 0 \\
-\beta_{S} \frac{\dot{\alpha}_{S}^{P}}{\alpha_{S}^{P}} & \geq 0 \\
\left(\beta_{S}-\bar{p}_{S e}+p_{S}\right) \frac{\dot{\bar{\rho}}_{S}}{\bar{\rho}_{S}} & \geq 0 \\
\operatorname{tr}\left[\left(\mathbf{T}_{S}^{\prime}-\overline{\mathbf{T}}_{S e}^{\prime}\right)^{t} \dot{\mathbf{F}}_{S}^{E}\left(\overline{\mathbf{F}}_{S}^{E}\right)^{-1}\right] & \geq 0
\end{aligned}
$$

where the third and fourth inequalities result from the partitioning of the stress with Eq. (99).

Using Eq. (124) we obtain a flow rule for $\dot{\alpha}_{S}^{P}$ :

$$
\frac{\dot{\alpha}_{S}^{P}}{\alpha_{S}^{P}}=-Y^{A}\left(\Lambda_{S}\right) \beta_{S},
$$

where $Y^{A}\left(\Lambda_{S}\right)$ is a positive scalar function of $\Lambda_{S}$. We also notice that the inequality Eq. (126) constrains only the deviatoric components of the stress. 
The pressure $\beta_{S}$ and the pressure $p_{S}$ are constrained by Eqs. (123) and (125). To satisfy these constraints let us assume the following constitutive relations:

$$
\begin{aligned}
& \beta_{S}=\beta_{S e}-\nu_{A} \frac{\dot{\alpha}_{S}^{E}}{\alpha_{S}^{E}}, \\
& p_{S}=\bar{p}_{S e}-\beta_{S}+\nu_{G} \frac{\dot{\bar{\rho}}_{S}}{\bar{\rho}_{S}} .
\end{aligned}
$$

The inequalities are satisfied if $\nu_{A}$ and $\nu_{G}$ are positive.

Two special cases are worth reviewing here. First, if we assume that $\nu_{G}=0$, then

$$
\frac{\dot{\alpha}_{S}^{E}}{\alpha_{S}^{E}}=\frac{1}{\nu_{A}}\left(\beta_{S e}-\bar{p}_{S e}+p_{G}\right), \quad \beta_{S}=\bar{p}_{S e}-p_{G}
$$

where Eq. (47) has been used. When we specialize our theory to a elastic solid and assume that $\alpha_{S}^{P} \equiv 1$, we obtain the type of relation used by Baer and Nunziato \{ see Eq. (59) in [3]) \}. Furthermore if we substitute Eq. (37) into this expression, we obtain

$$
\frac{\dot{\bar{\rho}}_{S}}{\overline{\bar{\rho}}_{S}}+\operatorname{div} \mathrm{v}_{S}=\frac{1}{\nu_{A}}\left(\beta_{S e}-\bar{p}_{S e}+p_{G}\right), \quad \beta_{S}=\bar{p}_{S e}-p_{G} .
$$

As an alternative approach suppose instead that $\nu_{G} \neq 0$ and $\nu_{A}=$ 0 , then

$$
\frac{\dot{\bar{\rho}}_{S}}{\bar{\rho}_{S}}=\frac{1}{\nu_{G}}\left(\beta_{S e}-\bar{p}_{S e}+p_{G}\right), \quad \beta_{S}=\beta_{S e} .
$$

Notice that this result is strikingly similar to Eq. (131). Indeed, in both cases the rate relations on the left are driven by the same combination of equilibrium pressures and only the presence of the term $\operatorname{div} \mathbf{v}_{S}$ differentiates the two rate relations. A more subtle difference between these two cases is that $\beta_{S}$ is constrained to its equilibrium value in one instance but not the other.

Baer and Nunziato use the term closure condition to describe relationships like Eqs. (131) and (132). They postulate a separate constitutive equation for the closure condition \{see Eq. (24) in [3])\}. While they claim to postulate a closure condition for the volume fraction, what they actually use is a closure condition for the distention. Our closure condition is Eq. (47):

$$
p_{S}=p_{G}
$$


Because we do not require $p_{S}$ to be an equilibrium pressure and because the distention gradient is a primitive variable we have obtained Baer and Nunziato's closure condition by postulating a simple constitutive equation for $\beta_{S}$, which is Eq. (128).

\subsection{Momentum and Energy Exchange}

Baer and Nunziato [3] prescribe specific expressions for momentum and energy exchange. We can obtain their relationships by prescribing

$$
\begin{aligned}
\tilde{\mathbf{m}}_{S}^{+} & =-\delta\left(\mathbf{v}_{S}-\mathbf{v}_{G}\right), \\
\tilde{e}_{S}^{+} & =-\delta \mathbf{v}_{S} \cdot\left(\mathbf{v}_{S}-\mathbf{v}_{G}\right)+c_{S}^{+} H_{S}+h\left(\vartheta_{G}-\vartheta_{S}\right)+\varphi_{S}\left(\beta_{S}-\beta_{S e}\right) \frac{\dot{\alpha}_{S}^{E}}{\alpha_{S}^{E}}
\end{aligned}
$$

where $\delta$ and $h$ are constitutive constants. For the special case when the distention gradient is elastic $\alpha_{S}^{P}=1$ and when Eq. (130) holds, then substitution of Eqs. (134) into Eqs. (65) and (68) yields the momentum and energy expressions in [3]. Baer and Nunziato considered a granular explosive that detonates to produce a gas so that $c_{S}^{+}<0$. Notice that the previous relationships indicate that the enthalpy $H_{S}$ of the burned solid is transferred to the gas. It also places all of the dissipative work due to both pore collapse and material diffusion into the gas.

When these assumptions are substituted into Eq. (121) we obtain the following dissipation inequality:

$$
\begin{gathered}
\frac{\nu_{A} \varphi_{S}}{\vartheta_{G}}\left(\frac{\dot{\alpha}_{S}^{E}}{\alpha_{S}^{E}}\right)^{2}-\frac{\varphi_{S} \beta_{S}}{\vartheta_{S}} \frac{\dot{\alpha}_{S}^{P}}{\alpha_{S}^{P}} \\
+\frac{1}{\vartheta_{S}}\left\{\operatorname{tr}\left[\left(\mathbf{T}_{S}^{t}-\overline{\mathbf{T}}_{S e}^{t}-\varphi_{S} p_{S} \mathbf{1}\right) \dot{\mathbf{F}}_{S}\left(\overline{\mathbf{F}}_{S}^{E}\right)^{-1}\right]\right. \\
\left.+\operatorname{tr}\left[\mathbf{F}_{S}^{-1} \mathbf{T}_{S}^{t} \mathbf{F}_{S}\left(\overline{\mathbf{F}}_{S}^{P}\right)^{-1} \dot{\overline{\mathbf{F}}}_{S}^{P}\right]-\rho_{S} \frac{\partial \psi_{S}}{\partial d_{S}} \dot{d}_{S}\right\}-\frac{\mathbf{q}_{S} \cdot \mathbf{G}_{S}}{\vartheta_{S}^{2}}-\frac{\mathbf{q}_{G} \cdot \mathbf{G}_{G}}{\vartheta_{G}^{2}} \\
-c_{S}^{+}\left[\frac{1}{\vartheta_{G}}\left(H_{S}-H_{G}\right)+\eta_{G}-\eta_{S}\right]+\frac{h}{\vartheta_{G} \vartheta_{S}}\left(\vartheta_{G}-\vartheta_{S}\right)^{2} \\
+\frac{\delta}{\vartheta_{G}}\left(\mathbf{v}_{S}-\mathbf{v}_{G}\right)^{2} \geq 0 .
\end{gathered}
$$

If we also require that

$$
\delta>0, \quad h>0, \quad-\mathbf{q}_{\xi} \cdot \mathbf{G}_{\xi} \geq 0,
$$


then application of Eqs. (123)-(126) along with our rules for damage and plastic flow allow us to reduce the dissipation inequality to

$$
-c_{S}^{+}\left[\frac{1}{\vartheta_{G}}\left(H_{S}-H_{G}\right)+\eta_{G}-\eta_{S}\right] \geq 0
$$

which is a constraint condition on the constitutive postulate for $c_{S}^{+}$.

\subsection{Alternate Exchange Formulation}

The exchange functions for momentum and energy Eq. (134) are the constitutive assumptions proposed by Baer and Nunziato. The terms containing $\delta$ and $h$ are conventional expressions for viscous drag and heat transfer between the constituents. Baer and Nunziato included the terms containing $H_{S}$ and $\beta_{S}$ to boost the heating of the gas during the burning of the solid. The transfer of the enthalpy of the solid $H_{S}$ to the gas during a chemical reaction seems plausible; however, we would argue that the last term in their energy exchange should be omitted because it represents the work dissipated by the stress in the solid. This dissipation term should add heat directly to the solid and not the gas. As an alternative energy exchange function, let us replace Baer and Nunziato's energy exchange postulate with

$$
\tilde{e}_{S}^{+}=-\delta \mathbf{v}_{S} \cdot\left(\mathbf{v}_{S}-\mathbf{v}_{G}\right)+h\left(\vartheta_{G}-\vartheta_{S}\right) \text {. }
$$

This leads to the following dissipation inequality:

$$
-c_{S}^{+}\left(\frac{\mathcal{G}_{S}}{\vartheta_{S}}-\frac{\mathcal{G}_{G}}{\vartheta_{G}}\right) \geq 0 .
$$

Thus one appropriate constitutive postulate for the reaction rate would be

$$
c_{S}^{+}=-\left(\frac{\mathcal{G}_{S}}{\vartheta_{S}}-\frac{\mathcal{G}_{G}}{\vartheta_{G}}\right) \mathcal{F}_{C}\left(\Lambda_{M}\right)
$$

where $\mathcal{F}_{C}\left(\Lambda_{M}\right)$ is an arbitrary positive scalar function of $\Lambda_{M}$. Baer and Nunziato have pointed out that detonations of granular explosives exhibit strong pressure dependence. Thus we might assume that $\mathcal{F}_{C}\left(\Lambda_{M}\right)$ is proportional to $p_{S}=p_{G}$. It has also been pointed out that suddenly compressed granular explosives and propellants undergo heterogeneous heating at the contact points between individual granules. These regions are called hot spots. It is believed that understanding 
hot-spot mechanisms is the key to modeling combustion and detonation processes. An example of this type of model may be found in Dienes [15]. Such considerations can also be used to determine the form of the function $\mathcal{F}_{C}\left(\Lambda_{M}\right)$.

\subsection{Conclusions}

The kinematics of our mixture theory are built on the distention gradient $\mathbf{A}_{S}$. In our constitutive theory for the granular explosive we have required this tensor to be the product of a scalar and the identity tensor. Thus the determinant of $\mathbf{A}_{S}$, which is the distention $\alpha_{S}$, is sufficient to completely define $\mathbf{A}_{S}$. The distention $\alpha_{S}$, volume fraction $\varphi_{S}$, and mass exchange $c_{S}^{+}$are interrelated through the balance of mass Eq. (37)

$$
\frac{\dot{\alpha}_{S}}{\alpha_{S}}=\frac{c_{S}^{+}}{\rho_{S}}-\frac{\dot{\varphi}_{S}}{\varphi_{S}}
$$

Because of this relationship, we might suppose that theories that use volume fraction as a primitive variable are equivalent to theories that use distention as a primitive variable. However, this is not true, and we can illustrate some of the important differences by comparing our theory, which employs $\alpha_{S}$ as a primitive variable, to the theory of Baer and Nunziato [3], which uses $\varphi_{S}$.

Baer and Nunziato follow the lead of the primary references in one important respect, the reaction mass $c_{S}$ is not a primitive variable. Indeed, $c_{S}$ (as opposed to $c_{S}^{+}$) is only defined in the primary reference by Bedford and the author, and we do not use it as a primitive variable in our constitutive equations. The implication of this omission is that mass that is native to the constituent is indistinguishable from mass that has been lost or gained through mass exchange. This is a reasonable assumption, but it has some interesting consequences. In particular, as illustrated in Figure 2 we found that either the distention $\alpha_{S}$ or the distention gradient $\mathbf{A}_{S}$ must be included in the primitive variables to distinguish between a stretching process and a burning process.

In Section 8.10, we pointed out that Baer and Nunziato also recognized this distinction because they actually wrote a closure equation for distention and not for volume fraction. What has not been clear until now is that not only the closure equation but all of the other 
constitutive equations for the solid should be written in terms of distention. Indeed for Baer and Nunziato's theory this implies that the Helmholtz free energy for their solid should be a function of distention instead of volume fraction. This was not immediately apparent from their derivation because they only defined equilibrium pressures in their theory, and they postulated the closure equation as a separate condition. However, as we have demonstrated their closure condition is actually a constitutive assumption for the stress $\beta_{S}$. Thus a dependence of the closure condition on distention implies that the stress and the Helmholtz free energy should also depend on distention.

Because Baer and Nunziato have retained $\varphi_{S}$ instead of $\alpha_{S}$ in the Helmholtz energy description for their porous solid, differences exist between their formulation and ours. In particular Eq. (137) differs from that contained in Baer and Nunziato \{See Eqs. (34) and (42) in [3]\}. The difference is the term $T_{G} \beta_{S} / T_{S}$ in their Eq. (42).

Theories for immiscible mixtures have often been based on two variables - the true density and the volume fraction. However, we have shown that a simple decomposition of the motion provides us with a convenient definition for the distention gradient. The determinant of the distention gradient is called the distention, and at first it looks as if the distention is just the inverse of the volume fraction. However, we now know that the presence of chemical reactions decouples the volume fraction from the motion and the distention. While the volume fraction may be an appropriate primitive variable for some constitutive descriptions, we have shown that it is distention that plays the important role in many constitutive descriptions including those for granular explosives.

\section{Acknowledgment}

I wish to thank R. M. Brannon at Sandia National Laboratories for her thoughful comments concerning the polar decomposition of $A_{S}$. I also wish to thank T. G. Trucano and M. R. Baer at Sandia National Laboratories and A. Bedford at The University of Texas at Austin, for reviewing the manuscript. This work was supported by Sandia National Laboratories, which is a multiprogram laboratory operated by Sandia Corporation, a Lockheed Martin Company, for the United States Department of Energy under Contract DE-AC04-94AL85000. 


\section{A Microinertia}

The theories of the primary references each contain microinertia equations. Inclusion of microinertia alters the forms of Eqs. (45) and (46) as well as the equations of balance of momentum and conservation of energy. There have been very few applications of mixture theory in which microinertia has been employed. Perhaps the only convincing demonstration of its usefulness has been to model bubbly liquids (see [17], [18], and [19]). Here we compare the two ways that microinertia has been modeled in the primary references with particular emphasis on the application to the bubbly liquid problem.

\section{A.1 True-Density Microinertia}

In the primary reference of Drumheller and Bedford [6] the microinertia description is based upon the true density $\bar{\rho}_{\xi}$ of the $\xi$-th constituent. When a constant microinertia term is included, Eqs. (45) and (46) become

$$
\bar{\rho}_{\xi}^{2} I_{\xi} \ddot{\bar{\rho}}_{\xi}-\frac{\bar{\rho}_{\xi}}{\varphi_{\xi}} c_{\xi}^{+} I_{\xi} \dot{\bar{\rho}}_{\xi}=\lambda-p_{\xi}
$$

and

$$
\frac{\mu_{\xi} J_{\xi}}{\varphi_{\xi}}=\lambda
$$

(Later in this section we shall illustrate how the microinertia $I_{\xi}$ can be explicitly determined for a bubbly liquid.) The balance of momentum Eq. (54) becomes

$$
\rho_{\xi} \mathbf{a}_{\xi}=\operatorname{div}\left(\mathbf{T}_{\xi}-\varphi_{\xi} \lambda \mathbf{1}\right)+\rho_{\xi} \mathbf{f}_{\xi}+\mathbf{m}_{\xi}^{+}+\lambda \operatorname{grad} \varphi_{\xi}-c_{\xi}^{+} \mathbf{u}_{\xi},
$$

and the conservation of energy Eq. (57) becomes

$$
\begin{aligned}
\rho_{\xi} \dot{E}_{\xi}+ & c_{\xi}^{+}\left(E_{\xi}+\frac{\lambda}{\bar{\rho}_{\xi}}\right)=\operatorname{tr}\left(\mathbf{T}_{\xi}^{t} \mathcal{L}_{\xi}\right)+\varphi_{\xi} p_{\xi} \frac{\dot{\bar{\rho}}_{\xi}}{\bar{\rho}_{\xi}}-\operatorname{div} \mathbf{q}_{\xi}+\rho_{\xi} r_{\xi} \\
& +e_{\xi}^{+}-\mathbf{m}_{\xi}^{+} \cdot \mathbf{v}_{\xi}+c_{\xi}^{+}\left(\frac{1}{2} \mathbf{u}_{\xi} \cdot \mathbf{u}_{\xi}+\frac{1}{2} I_{\xi} \dot{\bar{\rho}}_{\xi}^{2}\right)
\end{aligned}
$$

In special cases where the $\gamma$-th constituent has no microinertia, $I_{\gamma}=0$, we find that

$$
\lambda=p_{\gamma}
$$


If $I_{\xi}=0$ for several constituents then the $p_{\xi}$ of those constituents are equal.

You should also notice that for mixtures where it is appropriate to assume that $\mathbf{T}_{\xi} \equiv \mathbf{0}$, we obtain

$$
\rho_{\xi} \mathbf{a}_{\xi}=-\varphi_{\xi} \operatorname{grad} \lambda+\rho_{\xi} \mathbf{f}_{\xi}+\mathbf{m}_{\xi}^{+}-c_{\xi}^{+} \mathbf{u}_{\xi},
$$

which is similar to Eq. (8).

\section{A.2 Volume-Fraction Microinertia}

In the primary reference of Passman, Nunziato, and Walsh [8] the microinertia description is based on the volume fraction variables. Here the microinertia equations (45) and (46) are replaced by

$$
\frac{\mu_{\xi} J_{\xi}}{\varphi_{\xi}}=p_{\xi}
$$

and

$$
\rho_{\xi} k_{\xi} \ddot{\varphi}_{\xi}-c_{\xi}^{+} k_{\xi} \dot{\varphi}_{\xi}=p_{\xi}-\lambda .
$$

The balance of momentum Eq. (54) becomes

$$
\rho_{\xi} \mathbf{a}_{\xi}=\operatorname{div}\left(\mathbf{T}_{\xi}-\varphi_{\xi} p_{\xi} \mathbf{1}\right)+\rho_{\xi} \mathbf{f}_{\xi}+\mathbf{m}_{\xi}^{+}+\lambda \operatorname{grad} \varphi_{\xi}-c_{\xi}^{+} \mathbf{u}_{\xi},
$$

and conservation of energy Eq. (59) becomes

$$
\begin{aligned}
\rho_{\xi} \dot{E}_{\xi}+ & c_{\xi}^{+} E_{\xi}=\operatorname{tr}\left[\left(\mathbf{T}_{\xi}^{t}-\varphi_{\xi} p_{\xi} \mathbf{1}\right) \mathcal{L}_{\xi}\right]-p_{\xi} \dot{\varphi}_{\xi}-\operatorname{div} \mathbf{q}_{\xi} \\
& +\rho_{\xi} r_{\xi}+e_{\xi}^{+}-\mathbf{m}_{\xi}^{+} \cdot \mathbf{v}_{\xi}+c_{\xi}^{+}\left(\frac{1}{2} \mathbf{u}_{\xi} \cdot \mathbf{u}_{\xi}+\frac{1}{2} k_{\xi} \dot{\varphi}_{\xi}^{2}\right)
\end{aligned}
$$

Because of Eq. (153), when diffusion is not allowed, $\mathbf{v}_{\xi}=\mathbf{v}$, then

$$
\lambda=\frac{1}{N} \sum_{\xi} p_{\xi}
$$

Recall that $N$ is the number of constituents in the mixture. Comparison of this result to Eq. (145) demonstrates one influence of the different types of microinertia.

Notice that if $\mathbf{T}_{\xi} \equiv \mathbf{0}$, then we obtain

$$
\rho_{\xi} \mathbf{a}_{\xi}=-\varphi_{\xi} \operatorname{grad} p_{\xi}-\left(p_{\xi}-\lambda\right) \operatorname{grad} \varphi_{\xi}+\rho_{\xi} \mathbf{f}_{\xi}+\mathbf{m}_{\xi}^{+}-c_{\xi}^{+} \mathbf{u}_{\xi},
$$


which is similar to Eq. (8) when the additional assumption of $k_{\xi}=0$ is made. This type of microinertia produces a buoyancy term whereas the previous microinertia $I_{\xi}$ does not.

Passman, Nunziato, and Walsh attach one more constraint to their theory. For a saturated mixture in which $\sum_{\xi} \varphi_{\xi}=1$ and in the absence of their"equilibrated stress terms, they require that

$$
\sum_{\xi} \rho_{\xi} k_{\xi} \dot{\varphi}_{\xi}=0
$$

In particular for a binary mixture in which diffusion is absent, $\mathbf{v}_{\xi}=\mathbf{v}$, this means that

$$
\rho_{1} k_{1}=\rho_{2} k_{2} \text {. }
$$

As we shall see, this will lead to some interesting consequences.

\section{A.3 Bubbly Liquids}

Experimental data for both linear-harmonic waves and nonlinear-transient waves propagating through bubbly liquids have been successfully modeled using a mixture theory with microinertia based on the true density (see [17], [18], and [19]). The bubbly liquid was treated as a mixture of discrete gas bubbles $\xi=G$ in a continuous liquid $\xi=$ $F$. Liquid-vapor systems were also studied. The experiments were conducted on dilute mixtures $\varphi_{G} \approx 0.01$ with small nearly spherical bubbles. The following microinertia term was derived to model these data:

$$
I_{G}=\frac{R_{0}^{2} \bar{\rho}_{F}}{3}\left(\frac{\bar{\rho}_{G 0}^{2}}{\bar{\rho}_{G}^{11}}\right)^{1 / 3}=\frac{R^{2} \bar{\rho}_{F}}{3 \bar{\rho}_{G}^{3}},
$$

where $R_{0}$ and $\bar{\rho}_{G 0}$ are constants representing the reference state of the bubbles. This expression was derived for a single bubble that does not interact with neighboring bubbles. The radii $R$ of the bubbles in a liquid suspension are given by

$$
R^{3} \bar{\rho}_{G}=R_{0}^{3} \bar{\rho}_{G 0}
$$


which is the true conservation of mass Eq. (35). ${ }^{10}$ It is to be understood that

$$
I_{F}=0, \quad k_{F}=0, \quad, k_{G}=0 .
$$

Recall that to facilitate the discussion we specialized Eq. (141) to the case of constant $I_{\xi}$. Thus $I_{G}$ cannot be substituted into Eq. (141); however, you can turn to [19] to see how we used the general microinertia equation to obtain

$$
R \ddot{R}+\frac{3}{2}\left(1-\frac{\varphi_{G}}{\varphi_{F}}\right) \dot{R}^{2}+\frac{\dot{\bar{\rho}}_{F}}{\bar{\rho}_{F}} R \dot{R}=\frac{p_{G}-p_{F}}{\bar{\rho}_{F}} .
$$

For the special case of a dilute system $\left(\varphi_{G} \rightarrow 0\right)$ in which the fluid density is constant, this microinertia equation reduces to the familiar Rayleigh-Plesset bubble equation.

Although this microinertia model for a bubbly liquid has been successfully applied to a variety of data, Passman, Nunziato, and Walsh [8] have criticized it for not exhibiting certain "properties." Even though it is clear that $I_{G}$ in Eq. (155) applies only to a dilute system, they state that $I_{G}$ should approach zero for two special types of bubbly liquids. First, in the limit of a very dilute suspension of virtually "no" bubbles, they claim that $I_{G}$ should have the following property:

$$
I_{G} \rightarrow 0, \text { as } \varphi_{G} \rightarrow 0 .
$$

As they correctly point out, our expression for $I_{G}$ meets this requirement because $R_{0} \rightarrow 0$ in this case.

Next they claim that $I_{G}$ should obey

$$
I_{G} \rightarrow 0, \text { as } \varphi_{G} \rightarrow 1 .
$$

In this case the bubbly liquid is "all" bubbles; that is, only gas. It could be argued that this is an unfair constraint on $I_{G}$ because it was derived for bubbly liquids and the individual bubbles coalesce and simply don't exist when they get too close together. Nevertheless, we do notice that in the case of all gas, the average density $\bar{\rho}_{F}$ of the fluid

\footnotetext{
${ }^{10}$ Equation 156 implies that we are ignoring mass exchange between the bubbles and the liquid. Mass exchange can be included by replacing Eq. (156) with

$$
\frac{4}{3} \pi R^{3} \bar{\rho}_{G}=\frac{4}{3} \pi R_{0}^{3}\left(\bar{\rho}_{G O}+c_{G}\right) .
$$
}


surrounding a bubble must approach zero. Thus our $I_{G}$ also meets this requirement. However, Passman, Nunziato, and Walsh claim that it does not (see page 318 of [8]). The reason for their erroneous claim is that they have misquoted our expression for $I_{G}$ in their Eq. (5C.8.15).

As we have stated, Passman, Nunziato, and Walsh hold the point of view that microinertia based on $\bar{\rho}_{\xi}$ is unnecessary. To defend their viewpoint they also formulate a model for a bubbly liquid. As we have shown, an obvious connection exists between the radius of a bubble and the true density $\bar{\rho}_{G}$ of the gas in the bubble. This is the true conservation of mass Eq. (156), which naturally lead us to formulate the Rayleigh-Plesset bubble equation using microinertia based upon $\bar{\rho}_{\xi}$. However, Passman, Nunziato, and Walsh do obtain a bubble equation using microinertia based on $\varphi_{\xi}$. How is this done?

They achieve their result by specializing their model to "no slip" and no chemical reactions. Thus $\mathbf{v}_{\xi} \equiv \mathbf{v}$ and $c_{\xi}^{+} \equiv 0$. Under these conditions, the conservation of mass Eq. (37) yields

$$
\dot{\varphi}_{G}\left(\frac{1}{\varphi_{G}}+\frac{1}{\varphi_{F}}\right)=\frac{\dot{\bar{\rho}}_{F}}{\bar{\rho}_{F}}-\frac{\dot{\bar{\rho}}_{G}}{\bar{\rho}_{G}}
$$

With the additional assumption that $\dot{\bar{\rho}}_{F} / \bar{\rho}_{F}$ is negligible with respect to $\dot{\bar{\rho}}_{G} / \bar{\rho}_{G}$, they establish a kinematical link between $\bar{\rho}_{G}$ and $\varphi_{G}$ and effectively eliminated the distinction between microinertia based on $\bar{\rho}_{\xi}$ and $\varphi_{\xi}$. Thus it is obvious that for this specious example where diffusion, fluid compressibility, and chemical reactions are ignored, they can relate the radius $R$ of the bubble to the volume fraction $\varphi_{G}$ and derive a bubble equation. On pages $312-313$ of [8] they claim to have derived Rayleigh's bubble equation by this method. However, there is an error in their derivation. Their last equation on page 312 for the microinertia should have a factor 4 in the denominator and not a factor 8 . When this error is corrected, they do not obtain Rayleigh's bubble equation.

Given that they specialized their model to establish a kinematical link between the true gas density and the volume fraction of the bubbles, it is surprising that they do not achieve the proper result. Closer inspection of their model reveals that the source of this discrepancy is Eq. (154), which requires that

$$
\rho_{G} k_{G}=\rho_{F} k_{F}
$$


This constraint that they imposed on $k_{\xi}$ effectively doubles the microinertia in the mixture. It is the source of the missing factor of 2 . Of course, they can correct their theory by dropping this condition, or by arbitrarily dividing the microinertia between the constituents. However, even when corrected, their theory still cannot model bubblyliquid systems with diffusion.

\section{References}

[1] A. Bedford and D. S. Drumheller. Theories of immiscible and structured mixtures. Int. J. of Engineering Science, 21:863-960, 1983.

[2] C. Truesdell. Rational Thermodynamics. Springer-Verlag, New York, 1984.

[3] M. R. Baer and J. W. Nunziato. A two-phase mixture theory for the deflagration-to-detonation transition ( $\mathrm{ddt}$ ) in reactive granular materials. Int. J. of Multiphase Flow, 12:861-889, 1986.

[4] A. Bedford and D. S. Drumheller. A variational theory of immiscible mixtures. Archive for Rational Mechanics and Analysis, 68:37-51, 1978.

[5] A. Bedford. Hamilton's Principle in Continuum Mechanics. Pitman Publishing Ltd., London, 1985.

[6] D. S. Drumheller and A. Bedford. A thermomechanical theory for reacting immiscible mixtures. Archive for Rational Mechanics and Analysis, 73:257-284, 1980.

[7] J. W. Nunziato and E. K. Walsh. On ideal multiphase mixtures with chemical reactions and diffusion. Archive for Rational Mechanics and Analysis, 73:285-311, 1980.

[8] S. L. Passman J. W. Nunziato and E. K. Walsh. A theory of multiphase mixtures. In Rational Thermodynamics, pages 286325, 1984. By C. Truesdell.

[9] R. D. Mindlin. Micro-structure in linear elasticity. Archive for Rational Mechanics and Analysis, 16:51-78, 1964.

[10] A. C. Eringen. In Mechanics of Generalized Continua, pages 1835, 1968. Edited by E. Kroner. 
[11] L.Davison A. L. Stevens and M. E. Kipp. Theory of spall damage accumulation in ductile metals. J. Mechanics and Physics of Solids, 25:11-28, 1977.

[12] D. S. Drumheller. Introduction to Wave Propagation in Nonlinear Fluids and Solids. Cambridge University Press, Cambridge, 1998.

[13] Reint de Boer and J. Bluhm. The volume fraction concept in the porous media theory. Zeitschrift fuer angewandte Mathematik und Mechanik (ZAMM), 77:563-577, 1997.

[14] W. Herrmann. Constitutive equations for the dynamic compaction of ductile porous materials. Journal of Applied Physics, $40: 2490-2499,1969$.

[15] J. K. Dienes. On reactive shear bands. Physics Letters A, 118:433-438, 1986.

[16] P. Embid and M. Baer. Mathematical analysis of a two-phase continuum mixture theory. Continuum Mechanics and Thermodynamics, 4:279-312, 1992.

[17] D. S. Drumheller and A. Bedford. A theory of bubbly liquids. $J$. Acoustical Society of America, 66:197-208, 1979.

[18] D. S. Drumheller and A. Bedford. A theory of liquids with vapor bubbles. J. Acoustical Society of America, 67:186-200, 1980.

[19] D. S. Drumheller M. E. Kipp and A. Bedford. Transient wave propagation in bubbly liquids. J. Fluid Mechanics, 119:347-365, 1982. 